\title{
I gruppi continui proiettivi semplicemente infiniti nello spazio ordinario.
}

\author{
(Del prof. Giulio Pittarelut, a Roma.)
}

$\mathrm{N}_{\text {elle opere del prof. LiE: }}$

Vorlesungen über Differentialgleichungen, etc., Torlesungen ïber continuiliche Gruppen, edite tutt'e due dal dottore SchefFers,

Theorie der Transformationsgruppen, edita in collaborazione del professore F. ExGEL,

si trovano numerosi esempi ed applicazioni di gruppi proiettivi nel piano e nello spazio. Ma nella III. ${ }^{e}$ Abtheilung del III. $^{\circ}$ Abschnitt di quest' ultima opera è detto espressamente, a pag. 179, non esser nelle vedute dell'Autore di trattare $\mathrm{i}$ gruppi proiettivi nello spazio a tre dimensioni in maniera cos̀̀ compiuta come nel piano. E poco dopo, a pag. 182, soggiunge l'Autore: " Se noi aves" simo già ridotte ai diversi tipi le trasformazioni infinitesimali dello spazio, " come già facemmo nel piano a pag. 85, sarebbe assai facile in generale di " trovare tutte le curre gobbe, le quali ammettano almeno una trasformazione " infinitesimale proiettiva, ecc."

Lo scopo della presente Memoria è appunto di risolvere il problema precedente, sperando cos̀̀ di recare una qualche contribuzione, benchè piccola, ad una teoria che, creata dal LIE co' suoi lavori, fornisce anche materia a corsi universitari come quello del Kr.EIN (semestre d'estate del 1893), e del nostro Cremona (anno scolastico corrente).

A luogo opportuno ed a pie' di pagina saranno citati altri lavori che abbiano attinenza col soggetto. 


\section{$\S 1$. Formole generali in coordinate omogenee.}

Una trasformazione infinitesimale proiettiva dello spazio a tre dimensioni in coordinate omogenee è definita dal sistema di equazioni differenziali:

$$
\frac{d x_{i}}{d t}=\sum_{k} a_{i k} x_{k} \quad(i, k=1,2,3,4)
$$

ovvero dal simbolo:

$$
X f=\sum_{i} \sum_{i} u_{i k} x_{k} \frac{i f}{\partial x_{i}},
$$

dove $f$ è una funzione qualunque delle coordinate.

Le equazioni finite del gruppo generato da quella trasformazione infinitesimale, ciò̀ le espressioni delle $x$ in funzione di $t$, sono gl' integrali del sistema (1) con le condizioni iniziali che per $t=0$ sia $x_{i}=x_{i}^{0}$, essendo $x_{i}^{0}$ le coordinate del punto iniziale $x^{0}$.

Le (1) dànno le variazioni delle coordinate sotto la forma:

$$
\partial x_{i}=\partial t \sum_{k} a_{i k} x_{k}
$$

mentre, per la funzione $f$,

$$
\partial f=\sum_{i} \frac{\partial f}{\partial x_{i}} \delta x_{i}=\delta t \sum_{i} \sum_{k_{i}} a_{i k} x_{k}=\delta t X f
$$

Sia ora:

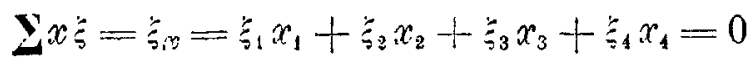

l'equazione di un piano $\xi$.

La trasformazione $X f$ applicata ai punti di questo piano li condurrà nei punti del piano $\zeta+\delta \zeta ;$ abbiamo cioè:

$$
(\xi+\partial \zeta)_{x+\partial x}=\xi_{x}+\zeta \partial x+x_{\partial y}+\partial \zeta_{\partial x}=0 .
$$

Trascurando l'ultimo termine del $2 .^{\circ}$ ordine, se vogliamo che tutta l'espressione precedente si riduca a $\succcurlyeq_{\infty}=0$, dobbiamo porre identicamente, qualunque sia $x$,

$$
\xi_{\delta x}+x_{\delta \xi} \equiv 0 \text {. }
$$

Ponendo per $\delta x_{i}$ i valori (3) possiamo scrivere:

$$
\sum_{i} \xi_{i} \sum_{k} \alpha_{i k} x_{k} \delta t+\sum_{i} x_{i} \delta \xi_{i} \equiv 0
$$


o sommando prima rispetto a $k$, poi rispetto ad $i$, abbiamo:

$$
\sum_{i} x_{i}\left(\delta \xi_{i}+\delta t \sum_{i} a_{k i} \xi_{k}\right) \equiv 0 \text {. }
$$

Onde si traggono le variazioni delle coordinate $\dddot{\leftrightarrow}_{i}$

$$
\delta \xi_{i}=-\delta t \sum_{i} a_{k i} \xi_{k}
$$

le equaziom differenziali

$$
\frac{d \dot{s}_{i}}{d t}=-\sum_{k} a_{k i} \grave{\zeta}_{k}
$$

ed il simbolo

$$
\Xi \varphi=-\sum_{i} \sum_{k} a_{k i} \varkappa_{k} \frac{\partial \varphi}{\partial \xi_{i}}
$$

I sistemi (1) e (6) di equazioni differenziali si dicono, nei trattati, sistemi aggiunti, e si ha la relazione:

$$
0=\mathbf{\Sigma} x_{i} \frac{d \xi_{i}}{d t}+\mathbf{\Sigma} \xi_{i} \frac{d x_{i}}{d t}=\mathbf{\Sigma} \frac{d}{d t}\left(\xi_{i} x_{i}\right), \quad \text { ossia } \quad \xi_{x}=0
$$

perche̊ $x$ e $\dot{\xi}$ si appartengono. I loro integrali dànno, in funzion di $t$, le coordinate dei punti della trajettoria di un punto iniziale $x^{0}$, ovvero le coordinate dei piani tangenti che, a partire da uno iniziale $\xi^{0}$, toccano una sviluppabile, osculatrice della trajettoria, se $\xi_{0}$ e $x_{0}$ si appartengono, come supponiamo.

Dinotiamo con $z_{i j}$ le coordinate di un raggio $x y$ e con $\zeta_{i j}$ quelle di un asse $\xi n$. Se per $y$ prendiamo il punto $x+\delta x$ e per $\eta$ il piano $\xi+\delta \xi$, abbiamo, a meno del fattore $\delta t$,

$$
\begin{aligned}
& z_{i j}=x_{i} \sum_{k} a_{j k} x_{k}-x_{j} \sum_{k} a_{i k} x_{k} \\
& \zeta_{i j}=\xi_{j} \sum_{k} a_{k i} \xi_{k}-\xi_{i} \sum_{k} a_{k j} \xi_{k} .
\end{aligned}
$$

Le rette (8) e (9) furon dette da ChLsLEs caratteristiche, rispettivamente, del punto $x$ e del piano $\xi$ nel movimento infinitamente piccolo d'un corpo solido libero nello spazio.

Si potrebbero anche assegnare le formole per la trasformazione infinitesimale dei raggi e degli assi partendo dalle trasformazioni dei punti e dei piani. Così al raggio $p+\delta p$ ed all' asse $\pi+\delta \pi$ spetteranno le coordinate che si traggono dalle due matrici

$$
\left\|\begin{array}{l}
x_{1}+\delta x_{1} \ldots x_{4}+\delta x_{4} \\
y_{1}+\delta y_{1} \ldots y_{4}+\delta y_{4}
\end{array}\right\|, \quad\left\|\begin{array}{l}
\xi_{1}+\delta \xi_{1} \ldots \xi_{4}+\delta \xi_{4} \\
\eta_{1}+\delta n_{1} \ldots n_{4}+\delta \eta_{4}
\end{array}\right\| .
$$


[Per non ingenerar confusione chiamerò $z 0 \%$ le caratteristiche e $p 0 \pi$ le rette qualunque $x y$ o $\xi_{\eta}$.]

Cosicchè, per esempio, trascurando gl' infinitesimi del $2 .^{\circ}$ ordine:

$$
p_{i j}+\delta p_{i j}=\left|\begin{array}{cc}
x_{i}+\delta x_{i} & x_{j}+\delta x_{j} \\
y_{i}+\delta y_{i} & y_{j}+\delta y_{j}
\end{array}\right|=p_{i j}+x_{i} \delta y_{j}-y_{i} \partial x_{j}-x_{j} \delta y_{i}+y_{j} \delta x_{i},
$$

onde poi

$$
\delta p_{i j}=x_{i} \delta y_{j}-y_{i} \delta x_{j}-x_{j} \partial^{2} y_{i}+y_{j} \partial_{i},
$$

e sostituendo alle variazioni delle coordinate le loro espressioni e passando alle equazioni differenziali, abbiamo un sistema di equazioni differenziali il cui tipo è:

$\frac{d p_{i j}}{d t}=a_{j 1} p_{i 1}-a_{i_{1}} p_{j_{1}}+a_{j 2} p_{i_{2}}-a_{i 2} p_{j_{2}}+a_{j_{3}} p_{i_{3}}-a_{i 3} p_{j 3}+a_{j_{4}} p_{i_{1}}-a_{i_{4}} p_{j 4} .\left(10^{\text {bis }}\right)$

Nei secondi membri di queste equazioni figureranno soltanto cinque delle 6 coordinate, mancherà cioè la coordinata $p_{h h}$ se $h k$ è una combinazione d'indici differenti da $i j$.

Si ottengono formole più semplici ed immediate partendo dalle coordinate $z_{i}(i=1, \ldots, 6)$ di KuEn legate dalla relazione fondamentale

$$
\sum_{i} z_{i}^{2}=z_{1}^{2}+z_{2}^{2}+z_{3}^{2}+z_{1}^{2}+z_{5}^{2}+z_{6}^{2} \text {. }
$$

Da questa segue per differenziazione:

$$
\sum_{i} z_{i} d z_{i}=z_{1} d z_{1}+\cdots+z_{6} d z_{6}=0
$$

Siano ora le formole di trasformazione infinitesimale proiettiva tra le $z$

$$
\frac{d z_{i}}{d t}=\sum_{k} \alpha_{i k} z_{k} \quad(i, k=1, \ldots, 6) .
$$

Dovendo esser verificata la (12) qualunque sia $z$, avremo l'equazione identica:

$$
y_{i} z_{i} \sum_{k} \alpha_{i k} z_{k}=\mathbf{y}_{i k} \alpha_{i k} z_{i} z_{k}=0 \text {. }
$$

Or questa equazione si deve ridurre alla (11), la sola identica esistente tra le coordinate di una retta qualunque dello spazio; dunque avremo:

per esempio, indi

$$
\alpha_{.14}=\alpha_{.22}=\cdots=\alpha_{.66}=\alpha,
$$

$$
\alpha_{i k}+\alpha_{k i}=0 \text {. }
$$

Da ciò si deduce che il determinante dei coefficienti è gobbo, ed il numero 
di essi da 36 si riduce a 16 , tanti quanti sono i coefficienti della trasformazione (1).

Se una funzione $\Omega$ delle coordinate di punti, di rette o di piani rimane invariata per la trasformazione, essa dovrà riprodursi moltiplicata per un fattore indipendente dalle coordinate. Sia, per esempio, $\Omega$ funzione di $x$ : si puo porre, a meno d'infinitesimi del $2 .^{\circ}$ ordine,

$$
\Omega(x+\delta x)=\Omega(x)+\sum_{i} \frac{\partial \Omega}{\partial x_{i}} \delta x_{i}
$$

Dorendo il secondo membro ridursi alla funzione $\Omega$, avremo, tenendo presente la $\left(3^{\text {bis }}\right)$, e dinotando con $\rho$ un fattore:

$$
\sum_{i} \frac{\partial \Omega}{\partial x_{i}} \delta x_{i}=\delta t X \Omega=\rho \Omega
$$

cosicchè allora viene appunto

$$
\Omega(x+\delta x)=(1+\rho) \Omega(x) .
$$

Ricordiamo poi che se un ente ammette la trasformazione infinitesimale $X f$ ammetterà tutte quelle del gruppo generato da $X f\left({ }^{*}\right)$.

Se la funzione $\Omega$ è il primo membro dell' equazione di un piano $\overbrace{}^{\prime}$ invariante dovremo avere qualunque sia $x$

$$
\rho \zeta_{x}=\left\{\xi_{1} \sum_{k} a_{i k} x_{k}+\cdots \mid \delta t\right. \text {. }
$$

Di qui ponendo $-\rho=\sigma \delta t$, avremo per le coordinate di un piano invariante $\xi$

$$
-\sigma \xi_{i}=\sum_{i} a_{k i} \xi_{k}\left({ }^{* *}\right) \text {. }
$$

E così per le coordinate di un punto invariante $x$

$$
s x_{i}=\sum_{i} \alpha_{i k} x_{k} \text {. }
$$

Le (17) e (18) forniscono una stessa equazione in $\sigma=(-s)$ di $4 .^{\circ}$ grado

$$
\Delta(\sigma)=\left|\begin{array}{llll}
a_{11}-\sigma & a_{12} & a_{13} & a_{14} \\
a_{21} & a_{22}-\sigma & a_{23} & a_{24} \\
a_{31} & a_{32} & a_{33}-\sigma & a_{34} \\
a_{41} & a_{42} & a_{43} & a_{44}-\sigma
\end{array}\right|=0 .
$$

(*) Vedasi: Lik, Vorlesü̈rgen ïber Differentialgleichungen, etc., pag. 72, Satz 9.

(*:) Il segno - e posto perchè così esso si presenta nella (6), ed è molto giovevole mantenerlo; come si vedrà sempre meglio in seguito. 
Consideriamo l'omografia tra due spazi $S$ ed $S$ ' sovrapposti definita dall' equazione [comesso $(1,1)]$

$$
\sum_{i k i k} a_{i k} x_{i}: z_{k}=0 \text {, }
$$

uvero separatamente in coordinate dj punti e di piani

$$
\begin{aligned}
s x_{i}^{\prime} & =\sum_{k}^{\mathbf{y}} a_{i k} x_{k} \\
-s_{i} & =\sum_{k} a_{k i} a_{k}^{\prime} .
\end{aligned}
$$

E notissimo allora che l'equazione $\Delta(\sigma)=0$ serve a determinare i punti ed i piani uniti dell'omografia (19).

Ad ogni tipo d'omografia (20) corrisponderà un tipo d'equazioni differenziali (1) formate sostituendo ad $s x_{i}^{\prime}$ le quantità $\frac{d x_{i}}{d t}$, ed in pari tempo ad ogui conseguente tipo duale d'omografia (21) corrisponderà un tipo d'equazioni differenziali (6) formate sostituendo a $\sigma \xi$ le quantità $\frac{d \xi^{\prime} i}{d t}$, e poi scrivendo $气$ in luogo di $\zeta^{\prime}$. Così facendo gl'integrali dei sistemi aggiunti (1) e (6) saranno sempre, senz'altro, determinati con la condizione $\xi_{x}=0$.

Il determinante $\Delta(\sigma)$ fu chiamato determinante caratteristico da $\mathrm{C}_{A \mathrm{u} H \mathrm{C}}$, ed è pur noto l'uso che si fa di esso per l'integrazione dei sistemi (1) e (6).

Si vede dunque che $i$ due problemi di classificare le omografia tra due spazi sovrapposti e d'integrare un sistema di equazioni differenziali lineari di $1 .^{\circ}$ ordine ed omogenee rientrano l'uno nell'altro.

Chiamerò l'omografia (19) aggiunta ai sistemi differenziali (1) e (6) c propriamente l'omografia (20) aggiunta al sistema (1) e l'omografia (21) aggiunta al sistema (6).

La classificazione delle omografie in uno spazio a un numero qualunque di dimensioni come teoria di forme bilineari si trova sostanzialmente nel lavoro fondamentale del Weierstrass: Zur Theorie der bilinearen und quadratischen Formen, Kön. Akad. zu Berlin, 1868. Ma la traduzione geometrica dei risultati analitici del prof. Weierstrass si deve al mio amico Segre, il quale dedico due Memorie al soggetto: l'una Sulla teoria e sulla classificazione delle omografie in uno spazio lineare ad un mumero qualunque di dimensioni, nel vol. 19, serie 3. ${ }^{\mathrm{a}}$ delle Memorie dei Lincei, 1884; l'altra Ricerche sulle omografie e sulle correlazioni in generale ecc. nel tom. 37, serie $2 .^{a}$ delle Memorie dell'Accademia di Torino, 1885. In quello stesso anno 1884 anche il prof. Loris 
nel vol. 22 del Giornale di Battiglin si occupò della teoria in quistione nella Nota: Sulle corrispondenze proiettive tra due piani e tra due spazi, dando una completa classificazione delle omografie.

Si comprende come partendo dalle formole di Weierstrass e mettendo a profitto i risultati geometrici, specialmente di SEGRE, io avrei potuto giungere a dar le forme canoniche dei secondi membri di (20) e (21) ovvero dei sistemi (1) e (6) per eseguirne poi l'integrazione. Ed io avevo cominciato a fare $i$ calcoli opportuni, allorchè il mio collega ed amico Castelncoro m'additò la Memoria del prof. Predella: Le omografie in uno spazio ad un numero qualunque di dimensioni, serie $2 .^{2}$, vol. 17, 1889, di questi Annali, nella quale la quistione è risoluta. Apprendesi anzi da quella Memoria che anche il Jordas nel tomo 73 dei Comptes Rendus a pag. 787 in unh Nota: Sur la résolution des équations différentielles linéaires, mostrò la possibilità di giungere ad equazioni del tipo

$$
-\frac{d y_{1}}{d t}=\sigma y_{1}, \quad \frac{d z_{1}}{d t}=\sigma z_{1}+y_{1}, \ldots \quad \frac{d w_{1}}{d t}=\sigma w_{1}+v_{1}
$$

dove $\sigma$ è radice multipla del determinante caratteristico annullante minori di dato ordine. Il risultato di JoRDAx 2 analiticamente importante; ma sono i lavori geometrici su riferiti quelli che permettono, come si vedrà, di delineare in un modo molto netto la posizione della curva integrale del sistema d'equazioni differenziali, non soltanto rispetto agli enti fondamentali dell'omografia, ma anche rispetto ad altri enti che si mutano in sè stessi per le trasformazioni del gruppo.

Perchè il lettore possa fare gli opportuni riscontri avverto ch'io adoprerò così il simbolo del Segre, ch'è in sostanza quello immaginato dal WeiLer (*), per indicare la distribuzione dei divisori elementari del determinante caratteristico, come il corrispondente del Predella. Per comprendere il simbolo adoperato dal Segre occorrerebbe un discorso un po' lungo; laddove il simbolo del Prederca è di più facile intelligenza. Il Predelda usa i numeri $0,1,2$ per dinotare un punto unito (dimensione 0), una retta unita (dimensione 1), un piano unito (dimensione 2). Se due enti si appartengono o coincidono $\mathrm{i}$ loro simboli saranno chiusi tra ( ). Per esempio il simbolo $\left[\left(\begin{array}{llll}0 & 0 & 0 & 0\end{array}\right]\right.$ indica l'esistenza di 4 punti uniti di cui 2 coincidenti; gli corrisponde il simbolo [2 11 1] di SEare che mostra due radici semplici ed una doppia del determi-

(*) Ueber clie verschiedenen Gathïngen der Complexe zweiten Grades. Math. Ann., Bd. 7. 
nante caratteristico che non ne annulla $i$ subdeterminanti. Ancora nel simbolo $\left[\begin{array}{lll}1 & 0 & 0\end{array}\right]$ di Prederda è messa in chiaro l'esistenza di un raggio unito e due punti uniti fuori di esso; a quel simbolo corrisponde l'altro di SEgRE [ $\left[\begin{array}{llll}1 & 1 & 1 & 1\end{array}\right]$ che dinota l'esistenza di due punti uniti forniti da due radici semplici del determinante $\Delta(\sigma)$ alle quali si riferisce il gruppo 11 , e l'esistenza di una radice doppia che per l'annullarsi dei subdeterminanti del $3 .^{\circ}$ ordine fornisce non più un punto unito doppio, ma infiniti situati sopra un raggio, al quale si riferisce il gruppo (1 1 ) ecc. Adoprero pure i simboli di WeILER, nella classificazione fatta per le coordinate di rette inodificati da $S_{E G R E}$ in ciò che questi usa le parentesi | invece delle [ ] ecc.

Notisi infine che le omografic definite dalle equazioni integrali arranno la stessa distribuzione degli elementi uniti dell'omografia aggiunta alle equazioni differenziali, ma le radici e gl' invarianti assoluti saranno funzioni espo. nenziali delle grandezze $\sigma_{i} t$, come mostrerà l'integrazione.

\section{§ 2. I 13 tipi di gruppi.}

A) Le trajetrorie sono curye sghembe (e dulluente).

N. 1: $\left[\begin{array}{llll}1 & 1 & 1 & 1\end{array}\right] \equiv\left[\begin{array}{llll}0 & 0 & 0 & 0\end{array}\right]:$

Omografie aggiunte $\left({ }^{*}\right)$ :

$$
\begin{aligned}
& x_{1}^{\prime}=\sigma_{1} x_{1}, \quad x_{2}^{\prime}=\sigma_{2} x_{2}, \quad x_{3}^{\prime}=\sigma_{3} x_{3}, \quad x_{4}^{\prime}=\sigma_{4} x_{4} \\
& -\overbrace{1}^{\prime}=\sigma_{1} \xi^{\prime}, \quad-\xi_{3}=\sigma_{2} \xi_{2}^{\prime}, \quad-\xi_{3}=\sigma_{3} \xi_{3}^{\prime}, \quad-\xi_{4}=\sigma_{4} \xi^{\prime}{ }_{4} \text {. }
\end{aligned}
$$

Le quattro radici sono distinte; si hanno quattro punti uniti e quattro piani uniti che formano un tetraedro, tre coppie di rette unite che ne sono gli spigoli.

Le equazioni differenziali si formano e s'integrano subito. Chiamando $x^{2}$ o $\xi^{0}$ il punto ed il piano iniziale si hanno le:

$$
\left.\begin{array}{llll}
x_{1}=x_{1}^{0} e_{1}^{\sigma_{1} t}, & x_{2}=x_{2}^{0} e^{\sigma_{3} t}, & x_{3}=x_{3}^{0} e^{\sigma_{3} t}, & x_{1}=x_{4}^{0} e^{\sigma_{1} t} \\
\xi_{1}=\xi_{1}^{0} e^{-\sigma_{1} t}, & \xi_{2}=\xi_{2}^{0} e^{-\sigma_{2} t}, \quad \xi_{3}=\xi_{3}^{0} e^{-\sigma_{3} t}, & \xi_{4}=\xi_{4}^{0} e^{-\sigma_{4} t},
\end{array}\right\}
$$

(*) Sopprimo il fattore di proporzionalità nelle $\alpha$, ma lo faccio eguale a -1 nelle $\xi$ per poter passare subito alla equazione differenziale $(6)$ del $\S 2$. 
come integrali dei sistemi

$$
\frac{d x_{i}}{d t}=\sigma_{i} x_{i}, \quad \frac{d \xi_{i}}{d t}=-\sigma_{i} \xi_{i}
$$

Bisogna poi distinguere due casi.

1." caso corrispondente al simbolo $\left\{\begin{array}{lllll}1 & 1 & 1 & 1 & 1\end{array} \mid\right.$, (caso generale).

Lue coordinate della caratteristica di $x$ e di quella di $\xi$ sono per le (2):

$$
z_{i j}=\left(\sigma_{j}-\sigma_{i}\right) x_{i} x_{j}, \quad \zeta_{i j}=\left(\sigma_{i}-\sigma_{j}\right) \xi_{i} \xi_{j} .
$$

Eliminando il prodotto $x_{1} x_{2} x_{3} x_{4}$ o l'altro $\xi_{1} \xi_{2} \xi_{3} \xi_{4}$ abbiamo le equazioni:

$$
\left.\begin{array}{l}
\frac{z_{12} z_{34}}{\left(\sigma_{1}-\sigma_{2}\right)\left(\sigma_{3}-\sigma_{4}\right)}=\frac{z_{23} z_{14}}{\left(\sigma_{2}-\sigma_{3}\right)\left(\sigma_{1}-\sigma_{4}\right)}=\frac{z_{31} z_{24}}{\left(\sigma_{3}-\sigma_{1}\right)\left(\sigma_{2}-\sigma_{4}\right)} \\
\frac{\zeta_{12} \zeta_{34}}{\left(\sigma_{1}-\sigma_{2}\right)\left(\sigma_{3}-\sigma_{4}\right)}=\frac{\zeta_{23} \zeta_{14}}{\left(\sigma_{2}-\sigma_{3}\right)\left(\sigma_{1}-\sigma_{4}\right)}=\frac{\zeta_{31} \zeta_{24}}{\left(\sigma_{3}-\sigma_{1}\right)\left(\sigma_{2}-\sigma_{4}\right)},
\end{array}\right\}
$$

che rappresentano, come è notissimo, il complesso tetraedrale di Reye. Il piano $\xi^{0}$, essendo osculatore alla trajettoria nel punto $x^{0}$, avıà le coordinate espresse da:

$$
\begin{aligned}
& \xi_{1}^{0}=-\left(\sigma_{2}-\sigma_{3}\right)\left(\sigma_{2}-\sigma_{4}\right)\left(\sigma_{3}-\sigma_{4}\right) x_{2}^{0} x_{3}^{0} x_{4}^{0}=\rho_{1} \frac{x_{1}^{0} x_{2}^{0} x_{3}^{0} x_{4}^{0}}{x_{1}^{0}} \\
& \xi_{2}^{0}=-\left(\sigma_{1}-\sigma_{3}\right)\left(\sigma_{1}-\sigma_{4}\right)\left(\sigma_{3}-\sigma_{4}\right) x_{1}^{0} x_{3}^{0} x_{4}^{0}=\rho_{2} \frac{x_{1}^{0} x_{2}^{0} x_{3}^{0} x_{4}^{0}}{x_{2}^{0}} \\
& \xi_{3}^{0}=\quad\left(\sigma_{1}-\sigma_{2}\right)\left(\sigma_{1}-\sigma_{4}\right)\left(\sigma_{2}-\sigma_{4}\right) x_{1}^{0} x_{2}^{0} x_{4}^{0}=\rho_{3} \frac{x_{1}^{0} x_{2}^{0} x_{3}^{0} x_{4}^{0}}{x_{3}^{0}} \\
& \xi_{4}^{0}=-\left(\sigma_{1}-\sigma_{2}\right)\left(\sigma_{1}-\sigma_{3}\right)\left(\sigma_{2}-\sigma_{3}\right) x_{1}^{0} x_{2}^{0} x_{3}^{0}=\rho_{4} \frac{x_{1}^{0} x_{2}^{0} x_{3}^{0} x_{4}^{0}}{x_{4}^{0}},
\end{aligned}
$$

dove $\rho_{i}$ sono $\mathrm{i}$ prodotti delle differenze di $\sigma_{h}, \sigma_{j}, \sigma_{k}$. Avremo dunque a meno del fattore $x_{1}^{0} x_{2}^{0} x_{3}^{0} x_{1}^{0}$, le

$$
\xi_{i}^{0} x_{i}^{0}=\rho_{i} .
$$

Ed allora le (1) dànno successivamente, in forza della (5),

$$
x_{i}=\frac{\hat{q}_{i}}{\xi_{i}^{0}} e^{\sigma_{i} t}=\frac{\rho_{i}}{\xi_{i}}
$$

che definiscono la ben nota reciprocita per la quale ad un piano a di equazione $a_{x}=0$ corrisponde la superficie di $3 .^{a}$ classe:

$$
\mathbf{\Sigma} \frac{\rho_{i} \alpha_{i}}{\zeta_{i}}=0,
$$


con quattro piani doppi, tangenti secondo una conica la superficie, nelle facce del tetraedro fondamentale; e ad un punto $a$ di equazione $a_{\xi}=0$ la superficie di $3 .^{\circ}$ ordine:

$$
\mathbf{y} \frac{p_{i} a_{i}}{x_{i}}=0
$$

con quattro punti doppi conici nei vertici dello stesso tetraedro.

Il complesso tetraedrale (4) dipende dalle sole $\sigma$; esso è invariante rispetto a tutte le trasformazioni del gruppo. Come prova di ciò si osservi che la trasformazione infinitesimale di una retta $p$ è data dalle formole:

$$
\frac{d p_{i j}}{d \cdot t}=\left(\sigma_{i}+\sigma_{j}\right) p_{i j} \text {. }
$$

Di qui segue, trascurando i termini del $2 .^{\circ}$ ordine:

$$
\left(p_{i j}+\delta p_{i j}\right)\left(p_{h k}+\delta p_{h k}\right)=p_{i j} p_{h k}+\left(\sigma_{i}+\sigma_{j}+\sigma_{h}+\sigma_{k}\right) p_{i j} p_{h k} \delta t
$$

onde se $i j, h l_{i}$ sono combinazioni binarie differenti la somma delle $\sigma$ è eguale a $\sigma_{1}+\sigma_{2}+\sigma_{3}+\sigma_{1}$, ed il secondo membro dell' equazione precedente si riduce al termine $p_{i j} p_{h k}$ moltiplicato pel fattore, che non muta, $1+\left(\sigma_{1}+\sigma_{2}+\sigma_{3}+\sigma_{4}\right) \partial t$.

Se invece, per un dato valor finito di $t$, cioè per una data trasformazione finita del gruppo, consideriamo come corrispondenti i due punti $x$ ed $x^{0}$ nei due spazi $S$ ed $S^{0}$ sovrapposti (e dualmente) trasformati l'uno dell'altro, rimettendo in vista nelle (1) il fattore di proporzionalità $\rho$, avremo le equazioni dell'omografia:

$$
\rho x_{1}=x_{1}^{0} e^{\sigma_{1} t}, \quad p x_{2}=x_{2}^{0} e_{2}^{\sigma_{2} t}, \quad \rho x_{3}=x_{3}^{0} e^{\sigma_{0} t}, \quad \rho x_{4}=x_{1}^{0} e^{\sigma}{ }_{4},
$$

ed $\mathrm{i}$ valori di $\rho$ che dànno gli elementi uniti, che sono poi i vertici (e le facce) dello stesso tetraedro, sono:

$$
\rho=e^{\sigma}{ }_{1}^{t}, \quad e^{\sigma_{2} t}, \quad e^{\sigma}{ }_{3}, \quad e^{s}{ }_{4} .
$$

Il determinante della sostituzione (8) è poi $e^{\left(\sigma_{1}+\sigma_{2}+\sigma_{3}+\sigma_{4}\right) t}$ sempre diverso da zero. Onde si conclude che l'omografia non è mai degenere, come osservò anche il Toresci nella sua Nota: Sul gruppo monomio individuato da una trasformazione infinitesimale proiettiva $\left(^{*}\right)$. E mentre gl' invarianti dell'omografia aggiunta sono $\mathrm{i}$ rapporti $\sigma_{1}: \sigma_{2}: \sigma_{3}: \sigma_{4}$, quelli della (8) sono invece $\mathrm{i}$ rapporti $e^{\sigma_{1} t}: e^{\sigma_{2} t}: e^{\sigma_{3} t}: e^{\sigma_{4} t}$.

(*) Rendiconti del Circolo matematico di Palermo, gennaio 1881; vedi unaltra Nota nei Rendiconti dell'Accad. di Napoli, maggio 1894: Sulle exuazioni fnite del grupo monomio, ecc. 
$\grave{E}$ noto pure come si compongano coi primi rapporti i rapporti anarmonici fondamentali dei quattro punti nei quali la caratteristica del punto $x$ incontra i quattro piani uniti (e dualmente). Perchè si ha per esempio, chiamando a uno dei suddetti rapporti anarmonici

$$
\alpha=\left(\begin{array}{llll}
1 & 2 & 3 & 4
\end{array}\right)=\frac{\sigma_{3}-\sigma_{3}}{\sigma_{2}-\sigma_{3}}: \frac{\sigma_{1}-\sigma_{4}}{\sigma_{2}-\sigma_{4}}=-\frac{\hat{\partial}_{31} z_{24}}{z_{33} z_{14}},
$$

per le (4) ecc. Inoltre poi il quoziente

$$
\frac{\sigma_{1}-\sigma_{3}}{\sigma_{2}-\sigma_{3}}
$$

è il rapporto anarmonico formato dal punto di contatto della tangente coi punti comuni alle tre facce $x_{1}=0, x_{2}=0, x_{3}=0$ del tetraedro fondamentale, ecc. $(*)$.

L'omografia (8) dà luogo invece ad un altro complesso di rette congiungenti coppie di punti corrispondenti come $x$ ed $x^{0}$. Le coordinate delle congiungenti e l'equazione del complesso si ottengono dalle (3) e (4) mutandori da per tutto le differenze $\sigma_{i}-\sigma_{j}$ nelle $e^{\sigma_{i} t}-e^{\sigma_{j} t}$.

Le equazioni (1) individuano famiglie di curve e di sviluppabili osculatrici diversissime, algebriche o trascendenti, che chiameremo curve o sviluppabili $W\left({ }^{* *}\right)$; ma è facile vedere come tutte si comportino rispetto agli elementi uniti della trasformazione.

Supponiamo $\sigma_{1}>\sigma_{3}>\sigma_{2}>\sigma_{1}$, e scriviamo le (1) sotto forma di rapporti come segue:

$$
x_{1}: x_{2}: x_{3}: x_{4}=x_{1}^{0}: x_{2}^{0} e^{\left(\sigma_{2}-\sigma_{2}\right) t}: x_{3}^{n} e^{\left(\sigma_{3}-\sigma_{1}\right) t}: x_{4}^{0} e^{\left(\sigma_{4}-\sigma_{1}\right) t}
$$

ovvero:

$$
x_{1}: x_{2}: x_{3}: x_{4}=x_{1}^{0} e^{\left(\sigma_{1}-\sigma_{4}\right) t}: x_{2}^{0} e^{\left(\sigma_{4}-\sigma_{4}\right) t}: x_{3}^{0} e^{\left(\sigma_{3}-\sigma_{4}\right) t}: x_{4}^{0} .
$$

Dalla prima forma di questi rapporti si vede che per $t=-\infty$ la curva passa pel punto $\xi_{1}=0$ di coordinate $(1,0,0,0)$, e dalla seconda si vede che per $t=\infty$ la curva passa pel punto $\xi_{4}=0$ di coordinate $(0,0,0,1)$.

(*) Vodi Lindwanx, Ueber unendlich kleine Bewegungen und über Kraftsysteme bei allgemeiner projeclivischer Massbestimmmung, nel vol. 7 dei Math. Annalen, Memoria che contiene numerosi ed importanti risultati, e che avró occasione di citare insieme alla prima parte del $20^{\circ}$ volume di Clebsch-Lindemann, Vorles. über clie Geometrie.

(*) W iniziale di «Wurf» (di STAUDT) quaderna d'elementi di dato rapporto anarmonico. Vedansi, per la ragion della denominazione, le Lezioni litogratate del prof. KLEIN, Göttingen, 1893, dal titolo: Einleitıng in die Köhere Geometrie $I$. 
Lo stesso ragionamento applicato alle $\zeta$ mostra che la curva è osculata, per $t=-\infty$, dal piano $x_{4}=-0$ nel punto $\zeta_{t}=0$, e per $t=\infty$ è osculata dal piano $x_{1}=0$ nel punto $\xi_{4}=0$. Esprimendo poi per mezzo delle (1) le coordinate $z_{i j}$ o $\zeta_{i j}$ della tangente in funzione di $t$, date dalle (3), si deduce allo

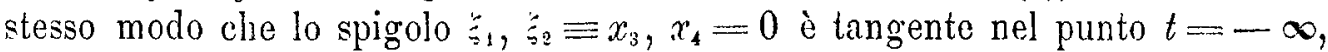
e lo spigolo $\zeta_{3}, \check{\zeta}_{4} \equiv x_{1}, x_{2}=0$ è tangente nel punto $t=\infty$.

Non supponendo relazione alcuna tra le $\sigma_{i}$, pur essendo queste distinte, nessuna quadrica e nessun complesso lineare è trasformato in sè stesso, come lo è invece il complesso tetraedrale più generale

$$
a z_{23} z_{14}+b z_{31} z_{94}+c z_{12} z_{34}=0 \text {, }
$$

la cui equazione in virtù della nota identità $z_{33} z_{14}+z_{31} z_{24}+z_{12} z_{31}=-0$ si può ridurre a forma binomia, per esempio, alla

$$
(b-a) z_{31} z_{94}+(c-a) z_{12} z_{34}=0 \text {. }
$$

2. ${ }^{\circ}$ caso comispondente al simbolo $\{\overline{(11)} 1111\}$.

Proponiamoci invece di determinare quelle quadriche e quei complessi lineari che, per certe relazioni tra le $\sigma$, possano trasformarsi in sè, e di trovare quelle relazioni. Se $F=a_{x}^{2} \dot{e}$, in notazione simbolica, una quadrica così fatta, applicando il criterio (16) del $\S 1$ dovremo avere l'identità

$$
a_{x} a_{l b c}=\rho a_{x}^{2},
$$

se per $d x_{i}$ si pongono i valori (2). Ciò porta a concludere che devono essere nulli i coefficienti $a_{i i}$ e due coppie di coefficienti $a_{h k}$ corrispondenti a due combinazioni $h k$ d'indici differenti (altrimenti le $\sigma$ dovrebbero essere eguali), e che supposta diverso da zero la coppia $\alpha_{14} a_{23}$ l'identità (9) che in questo caso si riduce alla

$$
a_{14}\left(\sigma_{1}+\sigma_{4}\right) x_{1} x_{4}+a_{23}\left(\sigma_{2}+\sigma_{3}\right) x_{2} x_{3}=p\left(a_{44} x_{1} x_{4}+a_{23} x_{2} x_{3}\right),
$$

porge la relazione tra le $\sigma$

$$
\sigma_{1}+\sigma_{4}=\sigma_{2}+\sigma_{3}(*) .
$$

In forza della relazione (10) le superficie del fascio-schiera

$$
F(x) \equiv x_{1} x_{4}+m x_{2} x_{3}=0 \quad \text { ovvero } \Phi(\hat{\xi}) \equiv \xi_{1} \dot{\xi}_{4}+\mu \xi_{2} \xi_{3}=0,
$$

(*) Si potrebbe supporre liversa da zoro li sola coppia di cocflicienti $a_{t i}$, $t_{h /}$ jol esempio $a_{33}, a_{12}$. In questo caso la quadrica é un cono $a_{33} x_{3}^{2}+2 a_{12} a_{1} x_{2}=0$, c dev'cssere $\sigma_{1}+\sigma_{2}=2 \sigma_{3}$. Questo caso sarà esaminato al $\$ 3$ in coordinate non omogenee. 
si mutano in sè stesse per tutte le trasformazioni del gruppo (1). Ma per le relazioni (7) e per la (10) anche il fascio di complessi lineari

$$
p_{44}+k p_{23}=0 \text { ovvero } \pi_{23}+k \pi_{14}=0\left(^{*}\right)
$$

si muta in sè, ed è il solo che abbia questa proprietà. Questo fascio di com. plessi ha per direttrici le rette unite $\xi_{1}, \xi_{1}=0, \xi_{2}, \xi_{3}=0$ che sono polari reciproche rispetto alle quadriche (11). E se $k=m$ le generatrici dell'iperboloide cui appartengono $亏_{1} \xi_{3}, \zeta_{2} \varkappa_{4}$ sono anche rette del complesso (12); se invece $k=-$ in apparterranno al complesso le rette del sistema $\xi_{1} \xi_{2}, \xi_{3} \xi_{4}$.

Applicando la trasformazione finita, per esempio alla quadrica (11), abbiamo, per (10),

$$
x_{1} x_{4}+m x_{2} x_{3}=e^{\left(\sigma_{1}+s_{4}\right) t}\left(x_{1}^{0} x_{4}^{0}+m x_{2}^{0} x_{3}^{0}\right),
$$

cioè $F(x)=e^{\left(\sigma_{1}+\sigma_{1}\right) t} F\left(x^{0}\right)$. Volendosi invece, dal punto di vista dell' algebra, $F(x)=F\left(x^{\prime}\right)$, bisognerebbe annullare l'esponente di $e$, qualunque sia $t$. Ciò richiede le due condizioni:

$$
\sigma_{1}+\sigma_{4}=0, \quad \sigma_{3}+\sigma_{3}=0 .
$$

Allora le radici dell' equazione che dà $i$ valori $\rho$ in (8) sono reciproche, come dev'essere per un teorema sulle forme quadratiche che si mutano in sè stesse dovuto al Frobenius (vedi Segre, Ricerche, ecc.). Or noi possiamo sempre soddisfare a quelle due condizioni. Infatti dalla (10) si ha:

$$
\sigma_{1}+\sigma_{i}=\sigma_{2}+\sigma_{3}=2 \sigma
$$

per esempio, onde:

$$
\left(\sigma_{1}-\sigma\right)+\left(\sigma_{1}-\sigma\right)=0, \quad\left(\sigma_{2}-\sigma\right)+\left(\sigma_{3}-\sigma\right)=0 .
$$

Scrivendo le (1) dopo l'introduzione del fattore di proporzionalità $e^{\text {st }}$, cos̀̀:

$$
x_{1}: x_{2}: x_{3}: x_{4}=x_{1}^{0} e^{\left(\sigma_{1}-\sigma\right) t}: x_{2}^{0} e^{\left(\sigma_{2}-\tau\right) t}: x_{3}^{0} e^{\left(\sigma_{3}-\sigma\right) t}: x_{4}^{0} e^{\left(\tau_{4}-\sigma\right) t}
$$

si vede ch'è lecito determinare $\sigma$ con le condizioni (14) concordanti in virtù di (10).

Ponendo $k=\frac{\sigma_{3}-\sigma_{2}}{\sigma_{4}-\sigma_{1}}$ ovvero $k=\frac{\sigma_{4}-\sigma_{1}}{\sigma_{3}-\sigma_{2}}$ si hanno quei due complessi che furon detti dal Linderyann nella Memoria citata (pag. 75-77) "Rotationscomplexe" e "Translationscomplexe" per quei movimenti dello spazio che,

(*) Caporadi e dex Puzzo, Introduzione alla teoria dello spazio rigalo, $\$ 20$, pag. 307 nelle Memorie di Geometria di Caporalt. 
in una determinazione metriea proiettiva, sono definiti come trasformazione in sè della quadrica fondamentale in modo che ciascun sistema di generatrici sia trasformato in sè stesso.

Le curve (1), situate allora sulle superficie $F(x)$ del fascio (11) contenenti i punti iniziali $x^{0}$ furon detti jvi dal Lindemann eliche proiettive $\left(^{*}\right)$. Esse sono le trasformate omografiche delle lossodromie della sfera.

Per provarlo consideriamo una qualunque delle quadriche $F(x)$ per es. quella passante pel punto $(1,1,1,1)$

$$
x_{1} x_{4}-x_{2} x_{3}=0 \text {. }
$$

Questa quadrica i proiettata stereograficamente dal punto su di essa, $\xi_{4}=0$, sopra un piano rappresentativo i cui punti $y$ li riferiamo a quel triangolo fondamentale ch'è sezione di esso piano coi piani $x_{1}=0, x_{2}=0, x_{3}=0$, mediante le formole note

$$
x_{1}: x_{2}: x_{3}: x_{1}=y_{1}: y_{2}: y_{3}: \frac{y_{2} y_{3}}{y_{1}}
$$

Sul piano rappresentativo l'omografia dello spazio individua un'omografia di simbolo $\left[\begin{array}{lll}0 & 0 & 0\end{array}\right]$, contenuta nella data, la cui espressione analitica è :

$$
y_{1}: y_{2}: y_{3}=y_{1}^{0} e_{1}^{\sigma_{1} t}: y_{2}^{0} e^{\sigma_{2} t}: y_{3}^{0} e^{\sigma_{3} t}
$$

e la curva rappresentata da queste equazioni è la proiezione stereografica della (1) situata sulla superficie (15). Supponiamo ora che la (15) sia una sfera, e che le (16) definiscano la proiezione stereografica in senso stretto, che sia cioè il piano rappresentativo parallelo al piano tangente in $\check{\varsigma}_{4}=0$ alla sfera. Su questo piano si ha in tal caso un'omografia della quale l'origine è un punto unito e gli altri due punti uniti sono i punti ciclici. Allora, com'è noto, le (17) definiscono una spirale logaritmica $\left(^{* *}\right)$, alle cui tangenti compete la proprietà (metrico-proiettiva) d'inclinarsi ad angolo costante sui raggi vettori. Proiettando di nuovo sulla sfera, il fascio dei raggi vettori dà il fascio dei meridiani passanti pel centro di proiezione, e la spirale logaritmica dà una

(*) Vedi anche: Vorles. «ber Geom., Bd. 2, th. 1.*, pag. 336 e seg.

(**) Clebsch, Vorles. viber die Geom. a pag. 979,988 e seg. nel capitolo sui connessi. Vedasi poi l'esteso e fondamentale lavoro dei prot., F. KLerv e S. LiE pubblicato nel vol. 4 dei Math. Annalen, 1871: Ueber diejenigen ebenen Curven uelche... in sich übergehen, oltro alle due Note del 6 e 13 griugno 1870 nei Comptes Rendus dal titolo: Sui une cortaine famille de courbes et de surfaces. - KreIs, Einleitung in die höhore Geometrie $I$, già citato. 
curva che, per la notissima proprietà della conservazione degli angoli nella proiezione stereografica, taglia sotto lo stesso angolo i meridiani che incontra: e questa curva è la lossodromia.

$3 .^{\circ}$ caso corrispondente al simbolo $\overline{(11)}(\overline{11}) 11$ !

Se anche la quadrica

0 il complesso

$$
x_{1} x_{2}+n x_{3} x_{4}=0 \text {, }
$$

$$
p_{12}+h p_{34}=0,
$$

dovessero mutarsi in sè stessi, sarebbe necessariamente

$$
\sigma_{1}+\sigma_{2}=\sigma_{3}+\sigma_{4} \text {. }
$$

Questa relazione insieme all'altra del caso precedente

fornisce

$$
\sigma_{1}+\sigma_{4}=\sigma_{2}+\sigma_{3},
$$

cioè :

$$
\sigma_{2}-\sigma_{4}=\sigma_{4}-\sigma_{2}
$$

onde poi

$$
\sigma_{2}=\sigma_{4}
$$

$$
\sigma_{1}=\sigma_{3}
$$

il determinante caratteristico $\Delta(\sigma)$ avrebbe due coppie di radici eguali. $\mathrm{Ma}$ questo caso sarà esaminato più tardi al n. ${ }^{\circ} 10$. Adunque nel gruppo [1 11111$]$ non vi sono trasformazioni corrispondenti al simbolo $\mid \overline{(11)}(\overline{11}) 11$.

Per realizzare un tipo cos̀̀ fatto bisogna considerare un'omografia qualunque, per esempio la

$$
x_{1}^{\prime}=\sigma_{1} x_{1}, \quad x_{2}^{\prime}=\sigma_{2} x_{2}, \quad x_{3}^{\prime}=\sigma_{3} x_{3}, \quad x_{4}^{\prime}=\sigma_{4} x_{4},
$$

ed in coordinate di rette

$$
p_{i j}^{\prime}=\sigma_{i} \sigma_{j} p_{i j} .
$$

La quadrica $x_{1} x_{4}+m x_{2} x_{3}=0$ ed il complesso $p_{14}+k p_{23}=0$ si mutano in sè stessi se

$$
\sigma_{1} \sigma_{4}=\sigma_{2} \sigma_{3} .
$$

E se lo stesso avviene per la quadrica $x_{1} x_{2}+n x_{3} x_{4}=0$ e pel complesso $p_{12}+h p_{34}=0$ dev'essere

$$
\sigma_{1} \sigma_{2}=\sigma_{3} \sigma_{4} \text {. }
$$

Da queste due relazioni tra le $\sigma$ segue moltiplicando membro a membro ovvero in croce

$$
\sigma_{1}^{2}=\sigma_{3}^{2}, \quad \sigma_{2}^{2}=\sigma_{1}^{2},
$$


onde, escluso il caso delle radici eguali,

$$
\sigma_{1}=-\sigma_{3}, \quad \sigma_{2}=-\sigma_{4} .
$$

Siccome (*) gl'invarianti assoluti o i rapporti delle radici sono i rapporti anarmonici che due punti corrispondenti qualunque formano coi punti nei quali la loro congiungente interseca $\mathrm{i}$ piani uniti, così chiamando $x x^{\prime}$ la coppia di punti corrispondenti ed $a_{1}, a_{2}, a_{3}, a_{4}$ i punti tracce della $x x^{\prime}$ sui piani uniti, abbiamo per le (19):

$$
\left.\begin{array}{ll}
\left(x x^{\prime} a_{1} a_{2}\right)=\frac{\sigma_{2}}{\sigma_{1}}, \quad\left(x x^{\prime} a_{1} a_{3}\right)=-1, \quad\left(x x^{\prime} a_{1} a_{4}\right)=-\frac{\sigma_{2}}{\sigma_{1}}, \\
\left(x x^{\prime} a_{2} a_{3}\right)=-\frac{\sigma_{1}}{\sigma_{2}}, \quad\left(x x^{\prime} a_{2} a_{4}\right)=-1, \quad\left(x x^{\prime} a_{3} a_{4}\right)=\frac{\sigma_{2}}{\sigma_{1}},
\end{array}\right\}
$$

non tutti indipendenti, ma dei quali si scorge il significato.

N. 2: $\left[\begin{array}{lll}2 & 1 & 1\end{array}\right] \equiv\left[\begin{array}{llll}0 & 0 & 0 & 0\end{array}\right], \sigma_{1}=\sigma_{2}$.

1. ${ }^{\circ}$ caso corrispondente al simbolo $\{2211\}$, , (caso generale).

Omografie aggiunte:

$$
\begin{aligned}
& x_{1}^{\prime}=\sigma_{2} x_{1}+\lambda_{1} x_{2}, \quad x_{2}^{\prime}=\sigma_{2} x_{2} \quad x_{3}^{\prime}=\sigma_{3} x_{3}, \quad x_{1}^{\prime}=\sigma_{4} x_{4} \\
& \left.-\xi_{1}=\sigma_{2} \xi_{1}^{\prime} \quad-\ddot{\xi}_{2}=\sigma_{2} \xi_{2}^{\prime}+\lambda_{1} \xi_{1},-\xi_{3}=\sigma_{3} \xi_{3}^{\prime},-\xi_{1}=\sigma_{1} \xi_{1}^{\prime}\right\}
\end{aligned}
$$

Gli elementi uniti sono:

il punto doppio $\xi_{1}=0$ ed il piano doppio $x_{2}=0$, doppi, perchè corrispondenti al valore $\sigma_{2}$ della radice doppia;

i punti ed i piani semplici

le rette doppie

$$
\begin{array}{ll}
\xi_{3}=0, & \xi_{4}=0, \\
x_{3}=0, & x_{4}=0 ;
\end{array}
$$

$$
\xi_{1} \cdot \xi_{3} \equiv x_{2} \cdot x_{4}, \quad \xi_{1} \cdot \xi_{4} \equiv x_{2} \cdot x_{3},
$$

aventi in comune il punto $\xi_{1}$ ed il piano $x_{2}$;

le rette semplici sghembe

$$
\xi_{1} \cdot \xi_{3} \equiv x_{3} \cdot x_{1}, \quad \xi_{3} \cdot \xi_{4}=x_{1} \cdot x_{2} .
$$

(*) Segre, Sulla teoria e sulla classifucazione, ecc., pag. 13 , 
Nelle formole dell'omografia aggiunta la costante $\lambda_{1}$ non può esser nulla (altrimenti si avrebbe il caso del n. $^{\circ} 6$ ), ma non è costante essenziale, dipende dal punto-unità del sistema di coordinate, come osserva il Predelca, e si può anche porre eguale ad 1 , come nelle formole di Jordan. Riferendosi infatti allo stesso tetraedro coordinato e mutando il punto-unità si potrà serivere $x_{i}=\alpha_{i} y_{i}$ e, conseguentemente, $x_{i}^{\prime}=\alpha_{i} y_{i}^{\prime}$, onde si avrà $y_{2}^{\prime}=\sigma_{2} y_{2}, y_{3}^{\prime}=\sigma_{3} y_{3}$, $y_{1}^{\prime}=\sigma_{1} y_{1}$, mentre la prima delle (1) darà $y_{1}^{\prime}=\sigma_{2} y_{1}+\lambda_{1} \frac{\alpha_{2}}{\gamma_{1}} y_{2}$. Nulla vieta di porre $\lambda_{1} \frac{\gamma_{s}}{\gamma_{1}}=1$ : ciò equivale, in sostanza, a scegliere in un modo o nell' altro il vertice $\xi_{2}=0$ sulla retta unita $\xi_{1} \xi_{0}$ ed il piano $x_{1}$ del fascio $\xi_{3} \xi_{4}$, vertice e piawo non uniti nell'omografia. Quest' osservazione valga per tutti i casi che, nel seguito, troveremo analoghi all'attuale.

Le equazioni differenziali sono:

$\begin{array}{llll}\frac{d x_{1}}{d t}=\tau_{2} x_{1}+\lambda_{1} x_{9}, & \frac{d x_{2}}{d t}=\tau_{2} x_{2}, & \frac{d x_{3}}{d t}=\tau_{3} x_{3}, & \frac{d x_{4}}{d t}=\tau_{4} x_{4} \\ \frac{d \xi_{1}}{d t}=-\tau_{2} \xi_{3}, & \frac{d \xi_{2}}{d t}=-\tau_{2} \xi_{2}-\lambda_{1} \xi_{1}, & \frac{d \xi_{3}}{d t}=-\tau_{3} \xi_{3}, & \frac{d \xi_{4}}{d t}=-\tau_{4} \xi_{4} \mid\end{array}$

Le ultime tre in $x_{2}, x_{3}, x_{4}$ s'integrano immediatamente; la prima, ponendovi il valor trovato di $r_{2}$ in franzione di $t$, diviene un'equazione lineare di primo ordine del noto tipo

$$
\frac{d y}{d t}=p y+q
$$

dove $p$ e $q$ sono funzioni di $t$, il cui integrale è il notissimo:

$$
y=e^{\int p d t}\left\{\int e^{-\int p d t} q d t+\operatorname{cost} .\right\} \text {. }
$$

Anche quest'osservazione valga fatta ora per sempre. Eseguendo le integrazioni abbiamo:

$$
\begin{aligned}
& x_{1}=\left(x_{1}^{0}+\lambda_{1} x_{2}^{0} t\right) e_{2}^{\sigma_{2} t}, \quad x_{2}=x_{2}^{0} e^{\sigma_{2} t}, \quad x_{3}=x_{3}^{0} e^{\sigma_{0} t}, \quad x_{1}=x_{4}^{0} e_{4}^{\sigma_{1} t} \text {, } \\
& \left.\xi_{1}=\xi_{1}^{0} e^{-\sigma_{2} t}, \quad \xi_{2}=\left(\xi_{2}^{0}-\lambda_{1} \xi_{1}^{0} t\right) e^{-\sigma_{2} t}, \quad \xi_{3}=\xi_{3}^{0} e^{-\sigma_{3} t}, \quad \xi_{1}=\xi_{1}^{0} e^{-\sigma_{4} t} .\right)
\end{aligned}
$$

Scrivendo le equazioni della prima linea sotto la forma:

$$
x_{1}: x_{2}: x_{3}: x_{1}=1: \frac{x_{2}^{0}}{x_{1}^{0}+\lambda_{1} x_{2}^{0} t}: \frac{x_{3}^{0} e^{\left(\sigma_{3}-\tau_{2} t\right.}}{x_{1}^{0}+\lambda_{1} x_{2}^{0} t}: \frac{x_{1}^{0} e^{\sigma_{4}-\tau_{2} t} t}{x_{1}^{0}+\lambda_{4} x_{2}^{0} t},
$$

Annali di Matematira, tomo XXII. 
posto $\sigma_{1}>\sigma_{3}>\tau_{2}$ ed osservando che:

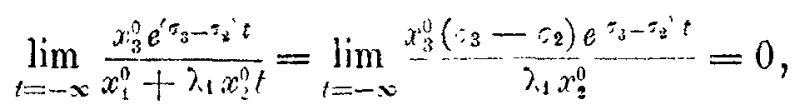

si trae che la curva passa pel punto $\vdots_{1}=0$ per $t=-\infty$. Così passa pel punto $\dot{\xi}_{1}=0$ corrispondentemente al valore $t=\infty$, ed inoltre in $\dot{s}_{1}=0$ è osculata dal piano $x_{1}=0$, in $\varkappa_{4}=0$ dal piano $x_{2}=0$, mentre la tangente nel primo

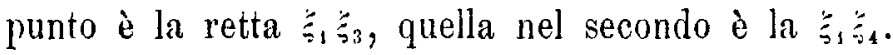

Formando le coordinate $z_{i j}$ della caratteristica di $x$ dalla matrice

$$
\| \begin{array}{cccc}
\sigma_{2} x_{1}+\lambda_{1} x_{2} & \sigma_{2} x_{2} & \sigma_{3} x_{3} & \sigma_{1} x_{4} \\
x_{1} & x_{2} & x_{3} & x_{4}
\end{array},
$$

si trova subito che l'equazione del complesso quadratico ì in questo caso:

$$
\lambda_{1}\left(\tau_{3}-\sigma_{1}\right) z_{23} z_{: 4}=\left(\tau_{2}-\sigma_{3}\right)\left(\tau_{2}-\tau_{1}\right) z_{12} z_{34} \text {. }
$$

Questo complesso corrisponde al simbolo [( 11 (1) (2 2)] nella classificazione di Wriler, pag. 188, n. ${ }^{\circ} 29$ della Memoria citata.

Fssendovi ancora tre piani uniti $x_{2} x_{3} x_{4}$ si può ricercare il rapporto anarmonico o, sulla tangente, del punto di contatto $x$ e delle tre tracee $a_{2} a_{3} a_{4}$ su quei piani; e si trova:

$$
a=\left(x a_{2} a_{3} a_{4}\right)=\frac{\sigma_{2}-\sigma_{3}}{\sigma_{2}-\sigma_{4}} .
$$

2. caso corrispondente al simbolo (11) 22 .

Se ci proponiamo di trovare le quadriche $F(x)$ o $\Phi(5)$ che si mutano in sè per le trasformazioni del gruppo, troviamo che $\mathrm{i}$ soli fasci-schiera di coni e di coniche

$$
\begin{aligned}
& x_{3} x_{4}+l x_{2}^{2}=0 \\
& \xi_{3} \xi_{1}+\lambda \xi_{1}^{2}=0,
\end{aligned}
$$

rispettivamente col vertice in $\breve{\zeta}_{1}=0$ e sul piano $x_{2}=0$, si mutano in sè stessi, quando però sia verificata la relazione

$$
2 \sigma_{2}=\sigma_{3}+\sigma_{1}
$$

Ed allora anche il fascio di complessi

$$
p_{12}+k p_{34}=0 \text { ovvero } \pi_{34}+k \pi_{12}=0,
$$

si muta in sè stesso. 
Determinando $l$ in modo che il cono (4) passi per $x^{2}$ e $i$ in modo che la conica (5) tocchi $5^{\circ}$, sarà la curva (3) posta sul cono e la sviluppabile osculatrice toccherà la conica.

N. $\left.3:\left[\begin{array}{ll}3 & 1\end{array}\right] \equiv\left[\begin{array}{llll}0 & 0 & 0\end{array}\right) 0\right],\left\{\begin{array}{ll}3 & 3\end{array}, \sigma_{3}=\sigma_{2}=\sigma_{1}\right.$.

Omografie aggiunte:

$$
\begin{aligned}
& x_{1}^{\prime}=\sigma_{1} x_{1}+\lambda_{1} x_{2}, \quad x_{2}^{\prime}=\sigma_{1} x_{2}+\lambda_{2} x_{3}, \quad x_{3}^{\prime}=\sigma_{1} x_{3}, \\
& x_{1}^{\prime}=\sigma_{4} x_{4} \text {, } \\
& -\xi_{1}=\sigma_{1} \xi_{1}^{\prime}, \quad-\xi_{2}=\sigma_{1} \xi_{3}^{\prime}+\lambda_{1} \xi_{1}^{\prime}, \quad-\xi_{3}=\pi_{1} \xi_{3}^{\prime}+\lambda_{2} \xi_{2}^{\prime}, \\
& -\check{\sigma}_{1}=\sigma_{1} \tilde{\sigma}_{1}^{\prime} \text {. }
\end{aligned}
$$

Gli elementi uniti sono:

il punto triplo $\varsigma_{1}=0$ ed il punto semplice $\tilde{5}_{4}=0$; il piano triplo $x_{3}=0$ ed il piano semplice $x_{4}=0$, le rette unite triple entrambe $\xi_{1} \cdot \xi_{4} \equiv x_{2} \cdot x_{3}$, $x_{3} \cdot x_{4} \equiv \xi_{1} \cdot s_{2} \cdot$

Integrando le equazioni differenziali

$$
\begin{aligned}
& \frac{d x_{1}}{d t}=\tau_{1} x_{1}+\lambda_{1} x_{2}, \quad \frac{d x_{2}}{d t}=\tau_{1} x_{2}+\lambda_{2} x_{3}, \quad \frac{d x_{3}}{d t}=\tau_{1} x_{3} \\
& \frac{d x_{4}}{d t}=\sigma_{4} x_{4}
\end{aligned}
$$

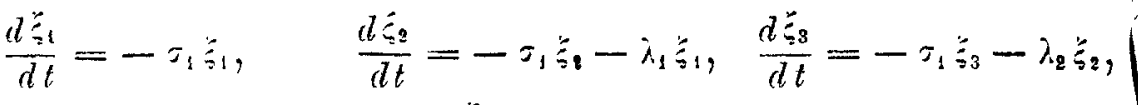

$$
\begin{aligned}
& \frac{d \dot{c}_{4}}{d t}=-\sigma_{1} \xi_{1}
\end{aligned}
$$

si trova:

$$
\begin{array}{ll}
x_{1}=\left(x_{1}^{0}+\lambda_{1} x_{2}^{0} t+\frac{1}{2} \lambda_{1} \lambda_{2} x_{3}^{0} t^{2}\right) e^{\sigma_{1} t}, & x_{2}=\left(x_{2}^{0}+\lambda_{2} x_{3}^{0} t\right) e^{\sigma_{1} t}, \\
x_{3}=x_{3}^{0} e^{\sigma_{1} t}, & x_{4}=x_{1}^{0} e^{\sigma} t, \\
\xi_{1}=\xi_{1}^{0} e^{-\sigma_{1} t}, & \xi_{2}=\left(\xi_{2}^{0}-\lambda_{1} \xi_{1}^{0} t\right) e^{-\sigma_{1} t}, \\
\xi_{3}=\left(\xi_{3}^{0}-\lambda_{2} \xi_{2}^{0} t+\frac{1}{2} \lambda_{1} \lambda_{2} \xi_{1}^{0} t^{2}\right) e^{-\sigma_{1} t}, & \xi_{4}=\xi_{4}^{0} e^{-\sigma_{1} t} .
\end{array}
$$

La trajettoria passa (e si vede come prima) pel punto $\check{s}_{1}$, corrispondente al valore $t=-\infty$, col piano osculatore $x_{1}$ e con la tangente $\xi_{1} \cdot \zeta_{2}$, e passa pel 
punto $\check{\zeta}_{4}$, corrispondente al valore $t=\infty$, col piano osculatore $x_{3}=0$ e con la tangente $\zeta_{1} \cdot$.

Il complesso delle caratteristiche $\dot{e}$ :

$$
\lambda_{1} z_{23} z_{24}+\lambda_{2} z_{34} z_{34}-\frac{1 . \lambda_{2}}{i_{1}-z_{3}} z_{2} z_{34}=0
$$

corrispendente al tipo [133)] di Weiser [pag. 200, n. 47$]$.

Non vi sono complessi lineari uniti (che si trasformano, civè, in sè stessi). Ma vi sono fasci uniti di coni e di coniche: essi sono, come si trora facilmente col solito metodo,

$$
\begin{aligned}
& F(x) \equiv \lambda_{1} x_{2}^{2}-2 \lambda_{2} x_{1} x_{3}+m x_{3}^{2}=0 ;
\end{aligned}
$$

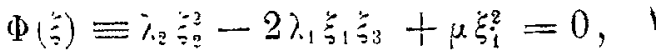

col vertice e col piano rispettivamente nel punto semplice e nol piano opposto $x_{4}=0$ : sono le sole quadriche che si trasformano in sè stesse.

Queste coniche sono le curve inviluppi delle loro tangenti, e quei coni sono i lunghi di rette, appartenenti alle omografie di simbolo [3] (ovvero $\left.\left[\begin{array}{ll}(0 & 0\end{array}\right)\right]$ ) del piano $x_{4}=0$ o del punto (stella) $\xi_{4}=0$ contenute nella omografia data. Le coordinate delle generatrici dei coni o delle tangenti alle coniche sono fornite dalle equazioni (3) toltene le espressioni di $x_{4}$ e di $\tilde{s}_{4}$, e sopprimendo, se si vuole, il fattore $e^{s_{1} t}$ che figura come fattore di proporzionalità: restano cos̀̀ le $x_{1}, x_{2}, x_{3} ; 亏_{1}, 亏_{2}, \check{亏}_{3}$ espresse con funzioni di $2 .^{\circ}$ grado nel parametro $t$. Inoltre poi $m$ e $\mu$ nelle (4) si possono determinare in modo whe il cono, o la conica, del fascio passi per $x^{0}$, o tochi ${ }^{0}$ : ed infatti, come verifica, sostituendo in $F(x)$ ed in $\Phi(\xi)$ i valori (3) si ha, a meno del fattore $e^{2 \sigma_{1} t} 0$ del fattore $e^{-2 \sigma_{1} t}$,

$$
F^{\prime}(x)=F\left(x^{0}\right)=0, \quad \Phi(\xi)=\Phi\left(\xi^{0}\right)=0,
$$

equazioni che determinano appunto $m$ e $\mu$.

I coni e le coniche dei fasci (4) hanno tra loro rispettivamente un contatto del $3 .^{\circ}$ ordine (quattro generatrici e quattro tangenti consecutive comuni), e propriamente $i$ coni lungo la retta unita $\vdots_{1} \cdot \overbrace{1} \equiv x_{2} \cdot x_{3}$ col piano tangente nel piano unito $x_{3}=0$ triplo, le coniche nell' altra retta unita $\varkappa_{1} \cdot \xi_{2} \equiv x_{3} \cdot x_{1}$ col punto di contatto nel punto unito triplo $\zeta_{1}=0$.

Determinando, come sopra, $m$ (e $\mu$ ) in modo che il cono passi per $x^{0}$ (e la conica tocchi $\xi^{0}$ ), la curva (3) giacerà sul cono (e la sviluppabile (3) toc- 
cherà la conica), anzi, come si vide poco fa, passerà pel vertice del cono stesso, avrà il piano osculatore $x_{3}=0$, e la tangente nella retta $\vdots_{1} \cdot \varkappa_{1}$, lungo la quale $i$ coni del fascio si sorrosculano.

Se, per fissar le idee, diamo a $t$ il significato di tempo, ricordando che per $t=\infty$ si ha il punto $\varkappa_{t}$ e per $t=-\infty$ si ha il punto il punto mobile descrive una curva (trascendente) sopra un cono quadrico, pas-

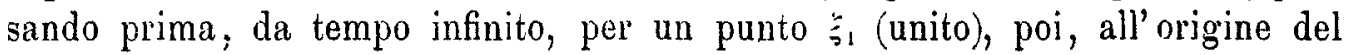
tempo, pel punto $x^{0}$, ed infine accostandosi vieppiù al vertice, dove giunge solo dopo trascorso un tempo infinito.

Volendo, possiamo determinare $\mu$, dato il cono $F(x)$ ciò̀ dato $m$, in modo che la conica $\Phi(\xi)$ sia, in coordinate di rette, la sezione prodotta dal piano $x_{4}=0$ sul cono $F(x)=0$. Per questo, ritenendo sempre che il covariante identico sia $\xi_{1} x_{1}+\xi_{2} x_{2}+\xi_{3} x_{3}+\xi_{4} x_{4}=0$ onde, nel piano $x_{4}=0$, $\xi_{1} x_{1}+\xi_{2} x_{2}+\xi_{2} x_{3}=0$, cercando l'equazione tangenziale di $F(x)$, si trova:

$$
\lambda_{3} \xi_{2}=2 \lambda_{1} \xi_{1} \xi_{3}-\frac{\lambda_{1} m}{\lambda_{2}} \xi_{1}^{2}=0
$$

e paragonando con $\Phi(\xi)$ si ha:

$$
\mu=-\frac{\lambda_{1} m}{\lambda_{2}}
$$

N. $\left.4:\left[\begin{array}{lll}2 & 2\end{array}\right] \equiv\left[\begin{array}{lll}(0 & 0\end{array}\right)\left(\begin{array}{ll}0 & 0\end{array}\right)\right], \tau_{2}=\tau_{1}, \tau_{1}=\tau_{3}$.

Omografie aggiunte:

$$
\begin{aligned}
& x_{1}^{\prime}=\sigma_{1} x_{1}+\lambda_{1} x_{2}, \quad x_{2}^{\prime}=\sigma_{1} x_{2}, \quad x_{3}^{\prime}=\sigma_{3} x_{3}+\lambda_{2} x_{4}, \\
& x_{1}^{\prime}=\sigma_{3} x_{4}
\end{aligned}
$$

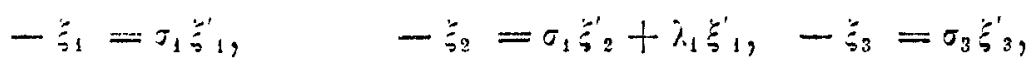

$$
\begin{aligned}
& -\breve{\zeta}_{1}=\sigma_{3} \dddot{\xi}_{4}+\lambda_{2} \check{\zeta}_{3} \text {. }
\end{aligned}
$$

Elementi uniti:

i punti doppi $\zeta_{1}==0, \breve{亏}_{3}=0$,

i piani doppi $x_{2}=0, x_{4}=0$,

le due rette unite sghembe $\xi_{1} \cdot \varkappa_{2} \equiv x_{3} \cdot x_{1}, \check{\xi}_{3} \cdot \varkappa_{1} \equiv x_{1} \cdot x_{2}$ giacenti sui piani uniti e passanti per i punti uniti,

un'altra retta che si appoggia alle precedenti ed è la retta $\overbrace{1} \cdot \overbrace{3} \equiv x_{2} \cdot x_{4}$ che congiunge $\mathrm{i}$ punti uniti ed è intersezione dei piani uniti. 
Equazioni differenziali:

$$
\begin{aligned}
& \frac{d x_{1}}{d t}=\tau_{1} x_{1}+\lambda_{1} x_{2}, \quad \frac{d x_{2}}{d t}=\tau_{1} x_{2}, \quad \frac{d x_{3}}{d t}=\tau_{3} x_{3}+\lambda_{2} x_{4}, \\
& \frac{d x_{4}}{d t}=\tau_{3} x_{4}
\end{aligned}
$$

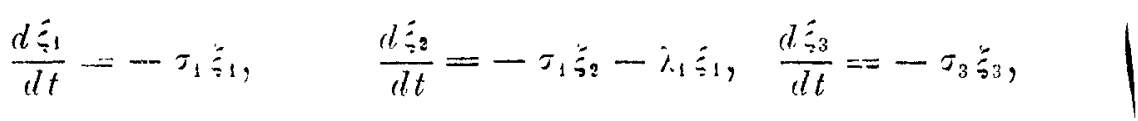

$$
\begin{aligned}
& \frac{d \dot{3}_{4}}{d t}=-\sigma_{3} \dot{\xi}_{4}-\lambda_{2} \dot{s}_{3} ;
\end{aligned}
$$

equazioni integrali:

$$
\begin{aligned}
& x_{1}=\left(x_{1}^{0}+i_{1} x_{2}^{0} t\right) e^{\sigma_{1} t}, \quad x_{2}=x_{2}^{0} e^{\pi_{1} t}, \quad x_{3}=\left(x_{3}^{0}+\lambda_{2} x_{4}^{0} t\right) e^{\pi_{0} t}, \\
& x_{4}=x_{4}^{0} e^{\sigma_{i} t}
\end{aligned}
$$

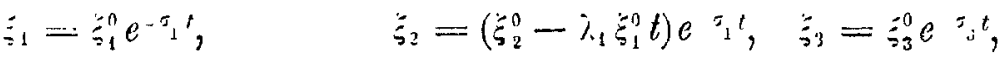

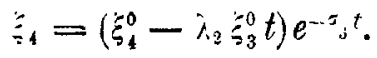

Supponendo, come sempre, $\sigma_{3}>\sigma_{1}$, si vede che la curra passa pel punto unito $\zeta_{1}=0$ dov' $\mathrm{e}$ oseulata dal piano unito $x_{4}=0$ ed ammette per tangente la retta unita $\xi_{1} \cdot \xi_{2} \equiv x_{2} \cdot x_{4}$ : ciò per $t=-\infty$. Invece per $t=\infty$ si ha il punto $\check{\zeta}_{3}=0$, il piano osculatore $x_{2}=0$ e la tangente $\check{\zeta}_{3} \cdot \zeta_{1} \equiv x_{1} \cdot x_{2}$.

Cosicchè l'asserzione del Lindemanx (Memoria citata, pag. 112) che la curva tocca la retta $x_{2}=x_{1}=0$ dev'esser corretta (nel Lindemann al posto di $x_{2}$ è $x_{1}$ ). Essa poggia sull' affermazione che per $t=-\infty$ il rapporto $x_{1}: x_{3}$ (nel Lindemans $x_{2}: x_{3}$ ) ha un valor finito, mentre $x_{3}$ ed $x_{4}$ svaniscono. Ciò non i vero, come si vede subito. Perchè, intanto, $x_{2}$ ed $x_{4}$ potrebbero per $t=-\boldsymbol{c}$ svanire soltanto allorchè fossero positive le due radici $\sigma_{1}$ e $\sigma_{3}$; ma nelle coordinate omogenee bisogna ragionar sui rapporti; e nel caso nostro, se $\sigma_{3}>\sigma_{1}$ il rapporto $\frac{x_{4}}{x_{2}}$ svanisce per $t=-\infty$. Poi il rapporto:

$$
\frac{x_{1}}{x_{3}}=\frac{x_{1}^{0}+\lambda_{1} x_{2}^{0} t}{x_{3}^{0}+\lambda_{3} x_{4}^{0} t} e^{\left(\sigma_{1} \ldots \sigma_{3}\right) t}=\frac{\frac{x_{1}^{0}}{t}+\lambda_{1} x_{2}^{0}}{\frac{x_{3}^{0}}{t}+\lambda_{2} x_{4}^{0}} e^{\left(\sigma_{1}-\sigma_{j}\right) t}
$$

per $t=-\infty$ essendo sempre $\sigma_{3}>\sigma_{1}$, diviene $\infty$. 
1l complesso delle caratteristiche è:

$$
\lambda_{1} \lambda_{2} z_{24}^{2}-\left(\sigma_{1}-\sigma_{3}\right)^{2} z_{12} z_{34}\left(^{*}\right) \text {. }
$$

Dalle (3) ricariamo le equazioni:

$$
\frac{x_{1}}{x_{2}}=\frac{x_{1}^{0}}{x_{2}^{3}}+i_{1} t, \quad \frac{x_{3}}{x_{1}}=\frac{x_{3}^{0}}{x_{4}^{0}}+i_{2} t,
$$

che rappresentano al variar di $t$ due fasci di piani proiettivi, le cui rette d'intersezione passano pel punto corrispondente al valor di $t$ della traiettoria e generano una quadrica rigata, che contiene la traiettoria suddetta. Gli assi dei fasci sono le rette unite sghembe $x_{1} \cdot x_{2}=0, x_{3} \cdot x_{4}=0$ dell'omografia; l'altra retta unita dell'omografia $x_{2} \cdot x_{4}=0$ corrispondente a $t= \pm \infty$ che passa anche pel punto $x^{0}$, è generatrice della quadrica.

Così pure le equazioni:

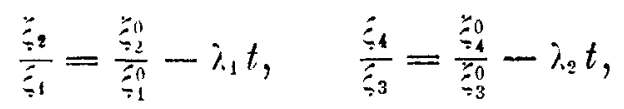

individuano due punteggiate proiettive cogli stessi assi sghembi precedenti;

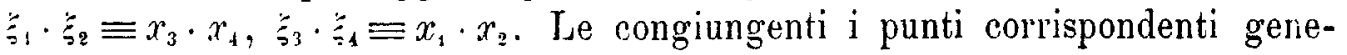
rano una quadrica rigata $i$ cui piani tangenti riescono osculatori alla sviluppabile fornita dalle (3), seconda linea orizzontale. La retta unita $\check{亏}_{1} \cdot \check{亏}_{3} \equiv x_{2} \cdot x_{4}$, corrispondente al valor $t= \pm \infty$, è anche generatrice di questa quadrica.

Le equazioni delle due quadriche sono:

$$
\begin{aligned}
& \frac{\lambda_{2} x_{1} x_{1}-\lambda_{1} x_{2} x_{3}}{x_{2} x_{4}}=\frac{\lambda_{2} x_{1}^{0} x_{1}^{0}-\lambda_{1} x_{2}^{0} x_{3}^{0}}{x_{2}^{0} x_{1}^{0}}=m \\
& \frac{\lambda_{2} \xi_{2} \xi_{3}-\lambda_{1} \xi_{1} \xi_{1}}{\zeta_{1} \xi_{3}}=\frac{\lambda_{2} \xi_{2} \xi_{3}^{0}-\lambda_{1} \xi_{1}^{0} \xi_{4}^{0}}{\zeta_{3}^{0} \xi_{3}^{0}}=\mu .
\end{aligned}
$$

Le (6) e (7) mostrano che $\mathrm{i}$ fasci-schiera di quadriche

$$
\begin{aligned}
& F(x) \equiv \lambda_{2} x_{1} x_{4}-\lambda_{1} x_{2} x_{3}-m x_{2} x_{4}=0 \\
& \Phi(\xi) \equiv \lambda_{2} \xi_{2} \xi_{3}-\lambda_{1} \xi_{1} \xi_{4}-\mu \xi_{1} \xi_{3}=0,
\end{aligned}
$$

sono quadriche invarianti pel gruppo di trasformazioni, e sono le sole, come si potrebbe trovare col solito metodo. Le $F$ e $\Phi$ rappresenteranno una stessa

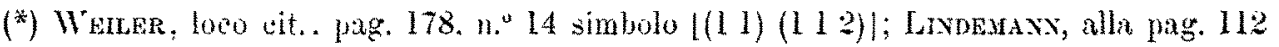
citatia nel testu. 
quadrica, in coordinate di punti o di piani, se $m=-\mu$. L'intersezione completa di due $F$ o due $\Phi$ si compone delle rette unite $\zeta_{1} \cdot \ddots_{3}, \ddots_{3} \cdot \ddots_{4}$ sghembe, e dell'altra $\varlimsup_{1} \overbrace{;}$ contata due rolte.

Ma r'è anche un fascio di conıplessi lineari che si trasforma in sè stesso ed ha pel equazione $\left(^{*}\right)$ :

$$
\lambda_{2} p_{14}-\lambda_{1} p_{23}+k p_{24}=0
$$

ovvero in coordinate $\pi_{i j}$

$$
\lambda_{2} \pi_{23}-\lambda_{1} \pi_{14}+\lambda_{21}=0
$$

le direttrici coincidenti del fascio sono date dalla retta unita dochè $l_{i}$ si pone eguale ad $m$ o a $\mu$ il complesso conterrà le generatrici di $F$ o di $\Phi$.

Tuito ciò che abbiamo detto fino qui corrisponde al simbolo $\{(\overline{31)} 11\}$; ma mulla invece corrisponde al simbolo $(\overline{(31)}(11) !$. Infatti il fascio di complessi aventi per direttrici le rette unite sghembe

$$
p_{12}+k p_{34}=0
$$

non è unito, nè può esserlo; perchè, dinotando con $p_{12}^{0}$ e $p_{34}^{0}$ due ralori iniziali, si ha $p_{12}=e^{\varepsilon_{1}{ }^{t}} p_{12}^{0}, p_{34}=e^{2 \sigma_{3} t} p_{31}^{0}$; permodoch̀̀

$$
p_{12}+k i p_{34}=e^{2 \pi}{ }_{1} t p_{12}^{0}+e^{25} t z_{i} p_{31}^{0} ;
$$

e dovrebbe poi essere $\tau_{1}=\tau_{3}$, il che non è. Ma, come si vide nel $3 .^{\circ}$ caso del $n .^{\circ} 1$, può bene avverarsi ciò per una trasformazione omografica qualunque (non di quelle che costituiscono il gruppo attuale) per esempio per la trasformazione aggiunta (1). Le quadriche (6) e (7) ed il complesso (8) sono enti invarianti anche rispetto ad essa; ma poiche si ha pure:

cosi :

$$
p_{1:}^{\prime}=\sigma_{1}^{2} p_{12}, \quad p_{34}^{\prime}=\sigma_{3}^{2} p_{34},
$$

$$
p_{12}^{\prime}+k p_{34}^{\prime}=\sigma_{1}^{2} p_{12}+\sigma_{3}^{2} k p_{34} .
$$

$\left({ }^{*}\right)$ La trasformazicne infinitesimale delle rette definita per mezzo delle $(10)$ del $\$ 1$ e delle (2) di questo numero è datia dalle formole:

$$
\begin{aligned}
& \frac{d p_{12}}{d l}=2 \sigma_{1} l_{12}, \quad \frac{d p_{34}}{d l}=2 \sigma_{33} l_{31}, \quad \frac{\left(l_{23}\right.}{d l}=\left(\sigma_{1}+\sigma_{3}\right) l_{23}+\lambda_{2} \mu_{24},
\end{aligned}
$$

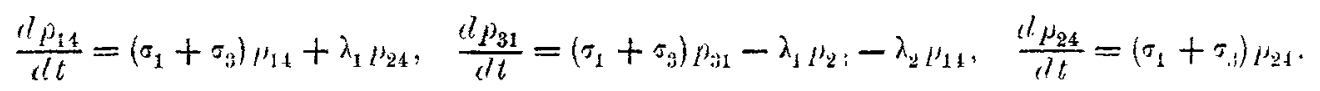

Applicando queste formole al un complesso lineares si deduce l'eyuazione (s) col solito metodo. 
Da ciò, per l'invariabilità del complesso, segue $\sigma_{1}^{2}=\sigma_{3}^{2}$ cioè, escludendo il caso di radici eguali, $\tau_{3}=-\tau_{1}$. Il significato geometrico più immediato di questo risultato si ha dalla relazione [vedi $n .^{0} 1$ equazione (20)]

$$
\left(x x^{\prime} a_{2} a_{4}\right)=-1 \text {. }
$$

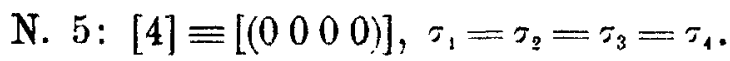

Omografie aggiunte:

$$
\left.\begin{array}{rl}
x_{1}^{\prime}=\sigma_{1} x_{1}+\lambda_{1} x_{2}, & x_{2}^{\prime}=\sigma_{1} x_{2}+\lambda_{2} x_{3}, \quad x_{3}^{\prime}=\sigma_{1} x_{2}+\lambda_{3} x_{4}, \\
-\dot{x}_{4}^{\prime}=\sigma_{1} x_{4} & =\sigma_{1} \xi_{1}^{\prime}, \quad-\xi_{2}=\sigma_{1} \xi_{2}^{\prime}+\lambda_{1} \xi_{4}^{\prime}, \quad-\xi_{3}=\sigma_{1} \xi_{3}^{\prime}+\lambda_{2} \xi_{2}^{\prime}, \\
-\xi_{4}=\sigma_{1} \xi_{4}^{\prime}+\lambda_{3} \xi_{3}^{\prime} . &
\end{array}\right\}
$$

Elementi uniti:

il punto quadruplo $弓_{1}=0$, il piano quadruplo $x_{4}=0$, e la retta $\varkappa_{1} \cdot \varkappa_{3} \equiv x_{3} \cdot$

Equazioni differenziali:

$$
\begin{aligned}
\frac{d x_{1}}{d t}=\sigma_{1} x_{1}+\lambda_{1} x_{2}, & \frac{d x_{2}}{d t}=\sigma_{1} x_{2}+\lambda_{2} x_{3}, \quad \frac{d x_{3}}{d t}=\sigma_{1} x_{3}+\lambda_{3} x_{1}, \\
\frac{d x_{4}}{d t} & =\sigma_{1} x_{1} \\
\frac{d \xi_{1}}{d t}=-\tau_{1} \xi_{1}, & \frac{d \xi_{2}}{d t}=-\sigma_{1} \xi_{2}-\lambda_{1} \xi_{1}, \quad \frac{d \xi_{3}}{d t}=-\sigma_{1} \xi_{3}-\lambda_{2} \xi_{2}, \\
\frac{d \xi_{4}}{d t} & =-\sigma_{1} \xi_{4}-\lambda_{3} \xi_{3} ;
\end{aligned}
$$

loro integrali:

$$
\left.\begin{array}{l}
x_{1}=\left(x_{1}^{0}+\lambda_{1} x_{2}^{0} t+\frac{1}{2} \lambda_{1} \lambda_{2} x_{3}^{0} t^{2}+\frac{1}{6} \lambda_{1} \lambda_{2} \lambda_{3} x_{1}^{0} t^{3}\right) e^{\sigma_{1} t}, \quad x_{3}=\left(x_{3}^{0}+\lambda_{3} x_{4}^{0} t\right) e^{\sigma_{1} t} \\
x_{2}=\left(x_{2}^{0}+\lambda_{2} x_{3}^{0} t+\frac{1}{2} \lambda_{2} \lambda_{3} x_{1}^{0} t^{2}\right) e^{\sigma_{1} t}, \\
\xi_{1}=\xi_{1}^{0}=x_{1}^{0} e^{\sigma_{1} t} \\
\xi_{3}=\left(\xi_{1}^{0} t, \quad \lambda_{2} \xi_{2}^{0} t+\frac{1}{2} \lambda_{1} \lambda_{2} \xi_{1}^{0} t^{0}\right) e^{-\sigma_{1} t} \\
\xi_{2}=\left(\xi_{2}^{0}-\lambda_{1} \xi_{1}^{0} t\right) e^{-\sigma_{1} t}, \quad \xi_{1}=\left(\xi_{1}^{0}-\lambda_{3} \xi_{3}^{0} t+\frac{1}{2} \lambda_{2} \lambda_{3} \xi_{2}^{0} t^{2}-\frac{1}{6} \lambda_{1} \lambda_{2} \lambda_{3} \xi_{1}^{0} t^{3}\right) e^{-\sigma_{1} t}
\end{array}\right\}
$$


Si pud prescindere, come si vede, dai fattori $e^{\tau_{1} t}, e^{-\sigma_{1} t}$ nelle espressioni delle $x$ e delle $\check{s}$.

La traiettoria è una cubica gobba, e l'jnviluppo è una sviluppabile di 3. ${ }^{a}$ classe duale della cubica, che sarì la sviluppabile osculatrice di questa, se $x^{0}$ e $\overbrace{0}^{0}$ si appartengono, come si suppone $\left(^{*}\right)$. La curva passa pel punto $\xi_{1}=0$ oscula il piano $x_{1}=0$ e tocca la retta $\varkappa_{1}^{\prime} \cdot x_{2}$, corrispondentemente al valore $t= \pm \infty$.

Le uniche quadriche che si trasformano in sè sono:

i coni del fascio

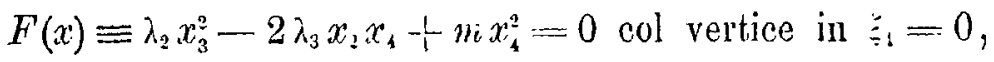

le coniche della schiera

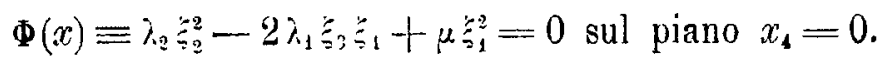

1 coni hamno un contatto del $3 .^{\circ}$ ordine sul piano unito $x_{4}=0$ lungo la generatrice $x_{3} \cdot x_{1} \equiv \zeta_{1} \cdot \zeta_{2} ;$ e così le coniche lo hamno nel punto $\zeta_{1}$ lungo la stessa retta $\xi_{1} \cdot \varkappa_{2} \equiv x_{3} \cdot x_{1}$. Questi coni (e queste coniche) sono gli enti invarianti nell'omografia della stella $\ddot{:}_{1}=0$ (e del piano $x_{1}=0$ ) contenuta nell'omografia data, di simbolo [3], cf: il n. ${ }^{\circ} 3$.

Inoltre determinando $m$ in modo che $F^{\prime}(x)$ passi per $x^{0}$, e $\mu$ in modo che $\Phi(\zeta)$ tocchi $\check{\sigma}^{\circ}$, il cono sarà quello che proietta la cubica gobba dal suo punto $\zeta_{1}=0$, e la conica sarà la sezione della sviluppabile osculatrice col suo piano $x_{1}=0$.

Il complesso delle caratteristiche è :

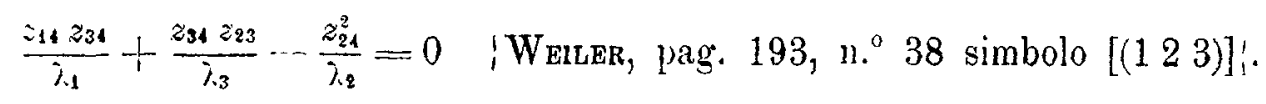

V'è inoltre un fascio di complessi lineari uniti, le cui direttrici coincidono

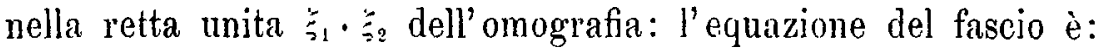

$$
i_{11} p_{23}-i_{2} p_{34}+l_{2} p_{34}=0 \text {. }
$$

(*) Dallequaziune del piano osculatore in un punto / si deducono lo conpdinate dial fiame osculatore $y_{3}^{0}$ nel punto iniziale . ${ }^{n}$ : csse sono:

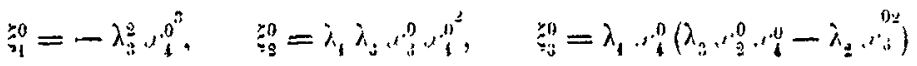

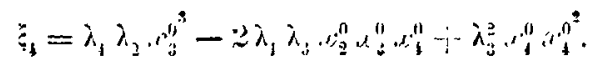

Esprasingi analogle si potreblern trovare prer tutti $i$ cosi. 
B) Le trajettorie sono curve piane (e dualmente).

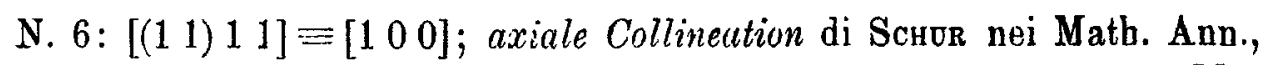
Bd. 19, pag. 430, prospettiva di $1{ }^{a}$ specie di Batragum nella terza Memoria: Sulla geometria projettiva.

Nella Memoria di SEgRe: Sulla teoria e sulla classificazione, ecc. è detto che questo è il caso generale di una seconda categoria di omografie, per le quali vi sarà sempre un raggio $r$ di punti uniti ed un asse $\rho$ di piani uniti, mentre nei cinque casi precedenti il numero degli elementi uniti era finito.

Ciò basta per concludere che le trajettorie sono curve piane (e le sviluppabili sono coni). Infatti se $x^{0}$ è il punto iniziale, il piano $x^{0} \cdot \rho$ è invariante perchè appartiene al fascio $o$. Applicando dunque ad $x^{0}$ una trasformazione qualunque del gruppo esso si sposterà sulla trajettoria, ma non lascerà il piano $x^{0} \cdot \rho$ : dunque la trajettoria ̀̀ piana $\left(^{*}\right)$.

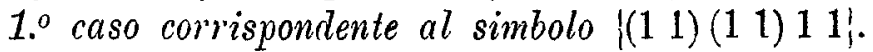

Per maggior brevità adotterò la segnatura $x_{i}^{\prime}$ in luogo della $\frac{d x_{i}}{d t}$, e così $\xi_{i}$ in luogo di $\frac{d \xi_{i}}{d t}$; allora l'equazione $x_{i}^{\prime}=\sigma_{i} x_{i}$ per esempio è un' equazione differenziale, ma la si può intendere anche come una delle equazioni dell'omografia aggiunta che serve a passare dallo spazio $S^{\prime}$ allo spazio $S^{\prime}$. Analogamente $\dddot{\leftrightarrow}_{i}^{\prime}=-\tau_{i} \check{s}_{i}$ ì un' equazione differenziale; ma se si vuole intenderla come equazione dell' omografia aggiunta, bisogna ricordare che in questa si

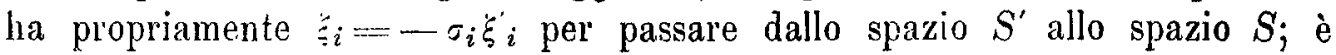
questo scambio di spazi che conviene tener presente per evitar dubbi.

Equazioni differenziali:

$$
\begin{array}{llll}
x_{1}^{\prime}=\sigma_{1} x_{1}, & x_{2}^{\prime}=\sigma_{1} x_{2}, & x_{3}^{\prime}=\sigma_{3} x_{3}, & x^{\prime}=\sigma_{4} x_{1}, \quad \\
\xi_{1}^{\prime}=-\sigma_{1} \xi_{1}, & \xi_{2}^{\prime}=-\sigma_{1} \xi_{2}, & \xi_{3}^{\prime}=-\sigma_{3} \xi_{3}, & \check{亏}_{4}^{\prime}=-\sigma_{4} \xi_{4} ; !
\end{array}
$$

integrali:

$$
\left.\begin{array}{llll}
x_{1}=x_{1}^{0} e^{\sigma_{1}} t, & x_{2}=x_{2}^{0} e^{\sigma_{1} t}, & x_{3}=x_{3}^{0} e^{\sigma_{3} t}, & x_{1}=x_{4}^{0} e^{\sigma_{4} t} \\
\xi_{1}=\xi_{1}^{0} e^{-\sigma_{1} t}, & \xi_{2}=\zeta_{2}^{0} e^{-\sigma_{1} t}, & \xi_{3}=\xi_{3}^{0} e^{-\sigma_{3} t}, & \zeta_{1}=\xi_{1}^{0} e^{-\sigma_{4} t} .
\end{array}\right)
$$

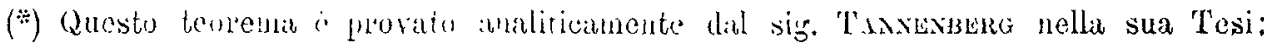

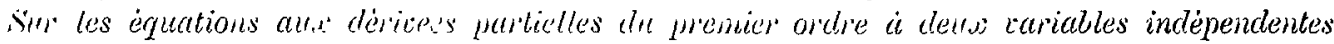
qui admettent un group' contime de transformations, citata nel $33^{\circ}$ vol. dell'opera di Lie-

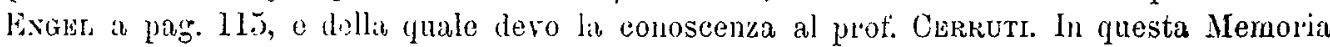
si trovano discussi cinçto tipi di trasformazioni omografiche intinitesinali insieme a cinqque corrispondenti categolio di curve golbse $\pi$. Cir. piii avanti alla fine del $\$ 3$. 
Gli elementi uniti sono:

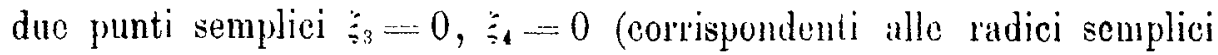

due piani semplici $x_{3}=0, x_{4}=0$;

$$
\left.\sigma_{3}, \sigma_{4}\right) \text {; }
$$

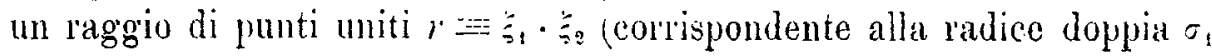

$$
\begin{array}{ll}
\equiv x_{3} \cdot x_{4} \quad \text { che annulla i minori del } \\
\quad 3 .^{\circ} \text { ordine); }
\end{array}
$$

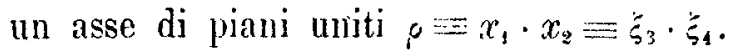

Conseguentemente sono uniti i fasci di centri $\check{\vdots}_{3}$ e di piani $x_{4}$ ed $x_{3}$ rispettivamente. Intorno a quei centri e su quei piani si hanno due omologie piane ordinarie aventi $r$ per asse di omologia e però di simboli [lll 11 l] 1 orvero $[10]$; mentre poi sui piani $x_{1}, x_{2}$ ed intorno ai punti $\zeta_{1}$, si hanno omografie ordinarie di simbolo [ $\left[\begin{array}{lll}1 & 1 & 1\end{array}\right]$ ovvero [ $\left[\begin{array}{lll}0 & 0 & 0\end{array}\right]$. Quelle omologie, appartenenti al gruppo, si hanno trascurando le terze o le quarte equazioni nelle due linee in (2); e quelle omografie si hanno invece trascurando le prime o le seconde. Si avverta pure che alle coppie $\xi_{1} \cdot \xi_{2}, x_{1} \cdot x_{2}$ di punti e di piani uniti possiamo sostituire un'altra coppia qualunque di punti su $r$ o di piani per $\rho$.

Le trajettorie sono curve piane situate nei piani $x_{2}^{0} x_{1}-x_{1}^{i} x_{2}=0$ del fascio o (al variar di $x^{3}$ ); e le sviluppabili sono coni coi vertici nei punti $\xi_{2}^{0} \xi_{1}-\xi_{1}^{0} \xi_{3}=0$ del raggio $r$ - Le caratteristiche dei punti $x$, come le congiungenti due punti corrispondenti qualunque, tagliano $\rho$; le caratteristiche dei piani 今, come le intersezioni di due piani corrispondenti qualunque, tagliano $r$.

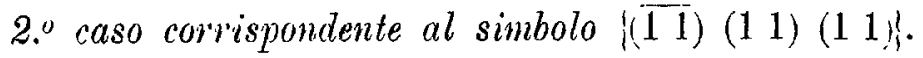

$\mathrm{Nel}$ caso precedente al fascio di complessi, avente por dretrici $r$ e $\rho$, di equazione

$$
p_{12}+k p_{34}=0,
$$

ed al fascio-schiera di quadriche, avente quelle rette per polari coniugate, di equazione

$$
\begin{aligned}
& x_{1} x_{2}+m x_{3} x_{4}=0 \\
& \xi_{3}+\mu \xi_{3} \xi_{1},
\end{aligned}
$$

corrispondono i due fasci:

$$
\begin{aligned}
& e^{2 \tau_{1} t} p_{12}^{0}+k e^{\left(\sigma_{3}+\sigma_{4}\right) t} p_{34}^{0}==0 \\
& e^{2 \sigma_{1} t} x_{1}^{0} x_{2}^{0}+m e^{\left(\pi_{3}+\sigma_{4}\right) t} x_{3}^{0} x_{4}^{0}=0
\end{aligned}
$$


Ma i fasci (3) e (4) si mutano in sè stessi se è verificata la condizione

$$
2 \sigma_{1}=\sigma_{3}+\sigma_{4} \text {. }
$$

Facendo un' analisi più accurata si troverà che non vi sono altri complessi uniti oltre a quelli del fascio (3); ma oltre al fascio (4) v'è un sistema lineare $\boldsymbol{c}^{3}$ di quadriche che, in forza della $(5)$, si muta in sè stesso, e che ha per equazione

o cosi pure

$$
\begin{aligned}
& F(x)=a_{11} x_{1}^{2}+a_{22} x_{2}^{2}+2 a_{12} x_{1} x_{3}+2 a_{34} x_{3} x_{4},
\end{aligned}
$$

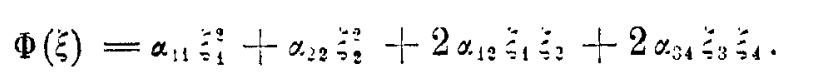

Potendo determinare uno dei cuefficienti $a$ o $\alpha$ in modo che $F(x)$ passi per $x^{\prime \prime}$ o $\Phi(\xi)$ tocchi $\check{\zeta}_{\circ}$, si rede subito che le trajettorie sono coniche situate su $F(x)$ e le sviluppabili sono coni circoscritti a $\Phi(\vdots)$.

La forma delle (6) mostra l'importante fatto che le rette $r$ e $\rho$ sono coniugate rispetto ad $F$ ed a $\Phi$. Basta per questo immaginar decomposto, per esempio, il trinomio $a_{11} x_{1}^{2}+a_{22} x_{3}^{2}+2 a_{12} x_{1} x_{2}$ nei suoi fattori lineari $p_{x}=p_{1} x_{1}$ $+p_{3} x_{2}$ e $q_{x}=q_{1} x_{1}+q_{2} x_{3}$, e poi scrivere $F$ così:

$$
F=p_{x} q_{x}+2 a_{34} x_{3} x_{4}=0
$$

donde si vede che, appunto, la retta $r \equiv x_{3} \cdot x_{1}$ è coniugata all'asse $\rho \equiv p_{\infty} \cdot q_{x}$ $\equiv x_{1} \cdot x_{2}$, che è lo stesso, poichè $\mathrm{i}$ piani $p_{x}=0, q_{x}=0$ formano fascio con $x_{1}, x_{2}$. Potendosi intendere che $\Phi(\xi)=0$ sia l'equazione tangenziale di $F\left({ }^{*}\right)$, vediamo subito come può avvenire il movimento: il punto $x$ percorre una conica di $F$, ed il piano $\xi$, tangente ad $F$ in $x$, inviluppa il cono circoscritto ad $F$ lungo quella conica, e se il piano di questa $\grave{e}$, come dev'essere, un piano per $\rho$, il vertice del cono sarà il polo del piano ossia un punto di $r$. Le posizioni iniziali saranno un punto $x^{0}$ di $F$ ed il piano tangente rispettivo $\xi^{\circ}$.

(*) L'entitione tungenziale di $F=0$ è:

$$
a_{22} \xi_{1}^{2}+a_{11} \xi_{32}^{2}-2 a_{12} \xi_{1} \xi_{2}+2 \frac{a_{11} a_{22}-a_{12}^{9}}{a_{34}} \xi_{34} \xi_{4}=0 ;
$$

di qui parrigonando coun $\mathbf{T}=0$ possiamo porre:

$$
\alpha_{11}=a_{22}, \quad \alpha_{i 2}=a_{11}, \quad \alpha_{12}=-a_{12}: \quad \alpha_{j 1}=\frac{a_{11} a_{22}-a_{12}^{*}}{a_{31}},
$$

londe, inverstmente.

$$
u_{11}=z_{22} . \quad a_{22,}=z_{11}, \quad \prime_{12}=-z_{12}, \quad u_{j 1}=\frac{z_{11} \alpha_{2,}-\alpha_{13}^{2}}{\alpha_{34}},
$$


Le equazioni (2) delle trajettorie e degli inviluppi si possono in questo caso semplificare.

Dalla (5) possiamo trarre:

$$
\sigma_{3}=\sigma_{1}+\sigma, \quad \sigma_{4}=\sigma_{1}-\sigma,
$$

onde, per esempio, dividendo per $e^{\sigma_{1}}$,

$$
x_{1}: x_{2}: x_{3}: x_{4}=x_{1}^{n}: x_{2}^{n}: x_{3}^{0} \epsilon^{5 t}: x_{4}^{0} e^{-5 t} \text {, }
$$

e posto $\lambda=e^{\text {tt }}$ e moltiplicando poi per $\lambda$

$$
\left.x_{1}: x_{2}: x_{3}: x_{4}=x_{1}^{0} \lambda: x_{2}^{0}\right\rangle: x_{3}^{n} \lambda^{2}: x_{4}^{n},
$$

che sono ben le equazioni di una conica.

S'intende già che in questo e nei seguenti casi il complesso quadratico delle caratteristiche si scinde nei due speciali aventi per direttrici o ed $r$; il primo formato dalle tangenti alle coniche, il secondo formato dalle generatrici dei coni.

N. 7: $\left.\left[\begin{array}{lll}(1 & 1) & 2\end{array}\right] \equiv\left[\begin{array}{lll}1 & (0 & 0\end{array}\right)\right]$.

Equazioni differcnziali:

$$
\begin{aligned}
& x_{1}^{\prime}=\sigma_{1} x_{1}, \quad x_{2}^{\prime}=\sigma_{1} x_{2}, \quad x_{3}=\sigma_{3} x_{3}+i_{1} x_{4}, \quad x_{4}^{\prime}=\sigma_{3} x_{4},
\end{aligned}
$$

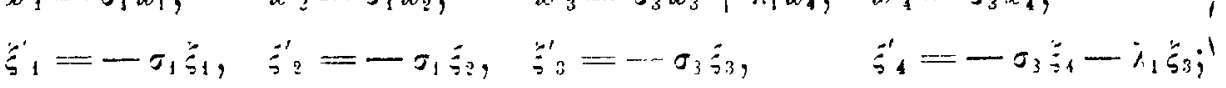

integrali :

$$
\begin{aligned}
& x_{1}=x_{1}^{0} e_{1}^{\sigma_{1} t}, \quad x_{2}=x_{2}^{0} e^{\sigma_{1} t}, \quad x_{3}=\left(x_{3}^{0}+\lambda_{1} x_{4}^{0} t\right) e^{\sigma_{3} t}, \quad x_{4}=x_{1}^{0} e^{\sigma_{3} t}
\end{aligned}
$$

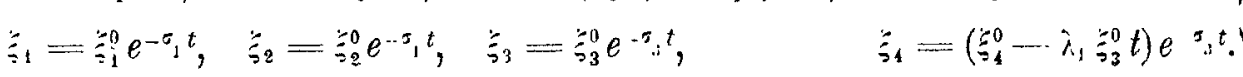

Gli elementi uniti sono:

un punto unito isolato $\check{亏}_{3}=0$,

un piano unito isolato $x_{4}=0$,

un raggio $r \equiv \zeta_{1} \cdot \zeta_{2} \equiv x_{3} \cdot x_{4}$ di punti uniti,

un asse $\rho \equiv x_{1} \cdot x_{2} \equiv \zeta_{3} \cdot \zeta_{1}$ di piani uniti.

Nel piano unito $x_{4}=0$ si ha un'omologia ordinaria $\left[\begin{array}{lll}1 & 1 & 1\end{array}\right]=\left[\begin{array}{ll}1 & 0\end{array}\right] \mathrm{di}$ centro $\zeta_{3}$ e di asse $r$ (e dualmente nella stella $\zeta_{3}=0$ si ha un'omologia ordinaria di piano $x_{4}=0$ sul quale giacciono sempre due rette corrispondenti e di asse $\rho$ intorno al quale si segano due piani corrispondenti). In ogni piano 
unito del fascio $\rho$, per esempio nel piano $x_{1}=0$ si ha un'omografia di simbolo $\left[\begin{array}{ll}2 & 1\end{array}\right] \equiv\left[\begin{array}{lll}(0 & 0 & 0\end{array}\right]$ col punto doppio $\check{5}_{3}=0$ unito, col punto unito semplice $\zeta_{2}$ ecc.

Le trajettorie sono curve trascendenti nei piani per $\rho$, le sviluppabili sono coni i cui vertici sono punti di $r$.

Non vi sono complessi lineari uniti (s'intende oltre ai complessi speciali $r$ e $\rho$ ) e le uniche quadriche unite sono le coppie di piani per $\rho$ e le coppie di punti su $r$.

Tutto ciò corrisponde al simbolo $(\overline{22}) 11$. Invece nel gruppo non si trovano omografie cui corrisponda il simbolo $(\overline{22}) \overline{11}$, per le quali, cioè sia unito il fascio dei complessi lineari avente per direttrici $r$ e $\rho$.

Infatti dall'equazione del fascio $p_{12}+k p_{31}=0$ si trae quella del fascio corrispondente:

$$
e^{2 \sigma} t{ }_{12}^{0}+k e^{25}{ }^{t} p_{34}^{0}=0 .
$$

E perchè questo fascio coincida col primo dev'essere $\tau_{1}=\sigma_{3}$; il che non è.

Ma anche qui come nel $n .^{\circ} 1$ e nel $n .^{\circ} 4$ cade in acconcio avvertire che un'omografia generale può ben presentare questo caso.

Sia l'omografia data dalle (1). Allora:

$$
p_{12}^{\prime}+k: p_{34}^{\prime} \equiv \sigma_{1}^{2} p_{12}+l_{i} \sigma_{3}^{2} p_{34} \text {. }
$$

Di qui, se il fascio è unito,

$$
\sigma_{1}=-\sigma_{3}
$$

che può ben essere. Siccome poi nell'omologia sul piano $x_{4}=0$ il rapporto $\sigma_{1}: \sigma_{3}$ è la costante dell'omologia, così nel caso della relazione (3) questa costante vale -1 , e l'omologia è involutoria.

Adunque cotale omologia si può presentare in un'omografia qualunque del tipo (1), ma giammai in quelle che appartengono al gruppo (2).

Un'altra definizione facile del rapporto $\sigma_{1}: \varsigma_{3}$ si trova agevolmente. La congiungente due punti corrispondenti $x$ ed $x^{\prime}$ nell'omografia si appoggia a $\rho$. Sia $a$ il punto di appoggio ed $a^{\prime}$ il punto comune ad $x x^{\prime}$ ed al piano unito $x_{4}=0$ : si ha:

$$
\left(x x^{\prime} a a^{\prime}\right)=\sigma_{3}: \sigma_{1} .
$$

$\mathrm{E}$, se ha luogo la (3), il gruppo $\left(x x^{\prime} a a^{\prime}\right)$ è armonico; onde $x x^{\prime}$ formano un' involuzione (i cui punti doppi sono $a a^{\prime}$ ) e sono permutabili tra loro. 


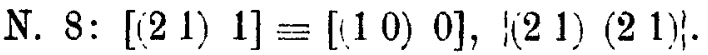

Equazioni differenziali:

$$
\begin{aligned}
& x_{1}^{\prime}=\sigma_{1} x_{1}+\lambda_{1} x_{3}, \quad x_{2}^{\prime}=\sigma_{1} x_{2}, \quad x_{3}^{\prime}=\sigma_{1} x_{3}, \quad x_{4}^{\prime}=\sigma_{4} x_{4}
\end{aligned}
$$

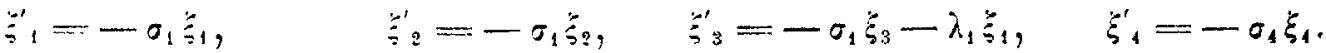

Gli elementi uniti sono:

un punto unito isolato $\dddot{\leftrightarrow}_{1}=0$,

un piano unito isolato $x_{1}=0$,

un raggio $r \equiv \xi_{1} \cdot \xi_{2} \equiv x_{3} \cdot x_{1}$ di punti uniti,

un asse $\rho \equiv x_{2} \cdot x_{3}=\zeta_{1} \cdot \varkappa_{4}$ di piani uniti,

ed $r$ e $\rho$ passano per $\xi_{1}$ e giacciono nel piano $x_{3}$.

Le trajettorie sono curve nei piani per l'asse $\rho$, e gl' inviluppi sono coni i sui vertici sono su $r$, curve ed inviluppo dati dalle equazioni integrali:

$$
\begin{aligned}
& x_{1}=\left(x_{1}^{0}+\lambda_{1} x_{3}^{0} t\right) e^{\sigma_{1} t}, \quad x_{2}=x_{2}^{0} e^{\tau_{1} t}, \quad x_{3}=x_{3}^{0} e^{\sigma_{1} t}, \quad x_{1}=x_{1}^{0} e^{\sigma_{4} t}
\end{aligned}
$$

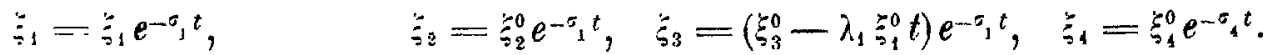

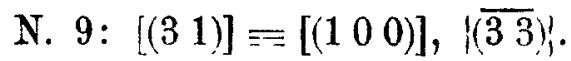

\section{Equazioni differenziali:}

$$
\begin{aligned}
& x_{1}^{\prime}=\sigma_{1} x_{1}+\lambda_{1} x_{3}, \quad x_{2}^{\prime}=\sigma_{1} x_{2}, \quad x_{3}^{\prime}=\sigma_{1} x_{3}+\lambda_{2} x_{1}, \quad, \\
& x_{1}^{\prime}=\sigma_{1} x_{1} \\
& \xi_{1}^{\prime}=-\sigma_{1} \xi_{1}, \quad \xi_{2}^{\prime}=-\sigma_{1} \xi_{2}, \quad \xi_{3}^{\prime}=-\sigma_{1} \xi_{3}-\lambda_{1} \xi_{1}, \quad \\
& \xi_{1}=-\sigma_{1} \xi_{1}-\lambda_{2} \xi_{3},
\end{aligned}
$$

integrali:

$$
\begin{gathered}
x_{1}=\left(x_{1}^{0}+\lambda_{1} x_{3}^{0} t+\frac{1}{2} \lambda_{1} \lambda_{2} x_{1}^{0} t^{2}\right) e^{\sigma_{1} t}, \quad x_{2}=x_{2}^{0} e^{\sigma_{1} t}, \quad x_{3}=\left(x_{3}^{0}+\lambda_{2} x_{1}^{0} t\right) e^{\sigma_{1} t}, \\
x_{1}=x_{4}^{0} e_{1}^{\sigma_{1} t} \\
\xi_{1}=\xi_{1}^{0} e^{-\sigma_{1} t}, \quad \xi_{2}=\xi_{2}^{0} e^{-\sigma_{1} t}, \quad \xi_{3}=\left(\xi_{3}^{0}-\lambda_{1} \xi_{1}^{0} t\right) e^{-\sigma_{1} t}, \\
\xi_{4}=\left(\xi_{4}^{0}-\lambda_{2} \xi_{3}^{0} t+\frac{1}{2} \lambda_{1} \lambda_{2} \xi_{1}^{0} t^{2}\right) e^{-\sigma_{1} t} .
\end{gathered}
$$

I fattori trascendenti $e^{\sigma}{ }^{t}, e^{-\sigma_{1} t}$ si possono sopprimere, e le (2) si riducono a funzioni di secondo grado del parametro $t$. Le trajettorie dunque sono 
coniche nei piani del fascio $\rho: x_{4}^{0} x_{2}-x_{2}^{0} x_{4}=0$, e gl' inviluppi sono coni àventi i punti sulla punteggiata $r: \xi_{2}^{0} \xi_{1}-\xi_{1}^{0} \xi_{2}=0$. I piani dell' asse $\rho$ ed i punti del raggio $r$, aventi a comune il punto $\xi_{1}=0$ ed il piano $x_{4}=0$, sono $\mathrm{i}$ soli elementi uniti dell'omografia.

Cerchiamo se vi sono quadriche che si trasformano in sè stesse.

Se la quadrica $F=a_{x}^{2}$ si deve trasformare in sè stessa, applicando il criterio solito, si trova che dev'essere identicamente:

$$
\frac{\partial F}{\partial x_{1}} \lambda_{1} x_{3}+\frac{\partial F}{\partial x_{3}} \lambda_{2} x_{4} \equiv 0
$$

Di qui le: $a_{11}, a_{12}, a_{13}, a_{23}, a_{34}=0, a_{14} \lambda_{1}+a_{33} \lambda_{2}=0$. Da quest'ultima si trae poi, supposto data la quadrica soggetta alle prime cinque condizioni, per i coefficienti della trasformazione:

$$
\lambda_{1}=\mu a_{33}, \quad \lambda_{2}=-\mu a_{14},
$$

essendo $\mu$ fattore non nullo, e non potendo essere nulli nè $\alpha_{33}$ nè $a_{14}$.

L'equazione della quadrica dunque è:

$$
F=a_{22} x_{3}^{2}+a_{33} x_{3}^{2}+a_{44} x_{4}^{2}+2 a_{14} x_{1} x_{4}+2 a_{24} x_{2} x_{4}=0,
$$

o anche, posto $\nu=\frac{1}{\mu}$, per le $(3)$

Analogumente:

$$
F=a_{22} x_{2}^{2}+a_{44} x_{4}^{2}+2 a_{24} x_{2} x_{4}+\nu\left(\lambda_{1} x_{3}^{2}-2 \lambda_{2} x_{1} x_{4}\right)=0 .
$$

$$
\Phi=\alpha_{22} \xi_{2}^{2}+\alpha_{11} \xi_{1}^{2}+2 \alpha_{12} \xi_{1} \xi_{2}+n\left(\lambda_{2} \xi_{3}^{2}-2 \lambda_{1} \xi_{1} \xi_{1}\right)\left(^{*}\right) . \quad
$$

La $F$ appartiene ad un fascio individuato dalla coppia di piani

$$
a_{22} x_{2}^{2}+a_{44} x_{4}^{2}+2 a_{24} x_{2} x_{4}=0,
$$

per l'asse $\rho$ e dal cono

$$
\lambda_{1} x_{3}^{2}-2 \lambda_{2} x_{1} x_{1}=0,
$$

(l'unico del fascio), forme ambedue invarianti.

(*) L'equazione tangonziale di $F$ e:

$$
a_{33}\left(a_{22} a_{44}-a_{24}^{9} \xi_{1}^{2}-a_{33} a_{44}^{2} \xi_{3}^{2}-a_{22} a_{14}^{9} \xi_{3}^{2}+2 a_{33} a_{14} a_{24} \xi_{1} \xi_{2}-2 a_{33} a_{22} a_{14} \xi_{1} \xi_{4}=0,\right.
$$

ch' è composta con le $\zeta$ come è composta la $\Phi$ : il che è ben naturale. 
Oltre al cono (5) appartiene al sistema lineare $\boldsymbol{\infty}^{3}$ di quadriche $F$ anche il cono:

$$
a_{41} x_{4}^{2}+\nu\left(\lambda_{1} x_{3}^{2}-2 \lambda_{2} x_{1} x_{4}\right)=0,
$$

ossia determinando il rapporto $a_{44}: \nu$ in modo che il cono passi pel punto $x^{0}$ :

$$
\frac{\lambda_{1} x_{3}^{2}-2 \lambda_{2} x_{1} x_{4}}{x_{4}^{2}}=\frac{\lambda_{1} x_{3}^{0^{2}}-2 \lambda_{2} x_{1}^{0} x_{4}^{0}}{x_{4}^{0^{0}}}\left(=-\frac{a_{44}}{v}\right) \text {. }
$$

Questo cono contiene la trajettoria del punto $x^{0}$ data dalle equazioni (2).

Il piano $r_{4}=0$, tangente alla giadrica $F$, la taglia nelle due rette fornite dalle equazioni:

$$
x_{4}=0, \quad a_{22} x_{2}^{2}+a_{33} x_{3}^{2}=0 .
$$

Or queste rette sono coniugate armoniche rispetto alla coppia

$$
\begin{aligned}
& x_{4}=0, \quad x_{2}=0 \quad \text { ciò̀ l'asse } \rho \\
& x_{4}=0, \quad x_{3}=0 \quad \text { ciò̀ il raggio } r .
\end{aligned}
$$

Dunque $\rho$ ed $r$ sono tangenti coniugate fisse rispetto a tutte le quadriche invarianti $F$.

Determinando le costanti di $F$ in modo ch'essa passi pel punto $x^{0}$, avremo dunque che la trajettoria di $x^{0}$ è la conica comune al piano del fascio $\rho$ : $x_{1}^{0} x_{2}-x_{2}^{0} x_{4}=0$ ed alla corrispondente superficie $F$. Per quella trajettoria poi passano $\infty^{2}$ superficie $F$, chè il passaggio per $x^{0}$ equivale ad una sola condizione lineare delle tre di cui si può disporre per le $F$. Considerando il cono $(6)$, o $\left(6^{\text {bis }}\right)$, che pur contiene la trajettoria in questione, facciasi variare il punto $x^{3}$ in modo che il piano $x_{4}^{0} x_{3}-x_{2}^{0} x_{4}=0$ non varii. Nasceranno altre trajettoric ed altri coni; ma come questi coni $\left[n .^{\circ} 3\right.$ equazione (4)] hanno tra loro un contatto del $3 .^{\circ}$ ordine lungo la generatrice $x_{3}=0, x_{4}=0$, cioè jungo $r$, così le coniche suddette hanno anch' esse un contatto del $3 .^{\circ}$ ordine nel punto $\xi_{1}=0$ con la tangente comune $p$.

Possiamo anche assoggettare le quadriche $F$ alla condizione che siano fisse per tutte la rette (7), il che richiede semplicemente

$$
\frac{a_{22}}{\nu_{1}}=\frac{a_{22}}{a_{33}}=\lambda=\text { costante. }
$$

Allora tutte le quadriche $F$ si toccano lungo quelle stesse rette fisse: le sfere che si toccano in un punto, per esemiro, sono in queste condizioni, e le rette comuni sono lo rette che dal punto di contatto vanno ai punti ciclici del piano tangente. 
Facciamo muovere adesso il piano intorno a $\rho$ e la trajettoria ch'esso contiene. Il polo del piano mobile percorrerà la retta $r$. E se la posizione iniziale $\xi^{0}$ del piano $\zeta$ è quella del piano tangente ad $F$ nel punto $x^{0}$, il piano $\zeta$ invilupperà il cono tangente ad $F$ lungo la trajettoria ed avente il vertice in quel punto di $r$ ch'è polo, rispetto ad $F$, del piano della trajettoria.

Avvengono dunque quì gli stessi movimenti che trovammo nel secondo caso del $n .{ }^{0}$ 6: soltanto qui non vi sono invarianti assoluti e le due rette $r$ e $p$ si tagliano.

Questo caso è trattato anch' esso dal Lindemann a pag. 112 della Memoria sulla Meccanica, e posteriormente a pag. 498 e seguenti delle Vorlesungen, 2. ${ }^{\text {" }}$ parte, a proposito della Geometria iperbolica. Le formole di questo Autore non sono per altro le canoniche adoperate da me; ma le si possono ridurre facilmente con sostituzioni lineari. Certo è, ad ogni modo, che specialmente il fatto che nell'omografia le rette $r$ e $\rho$ sono raggi ed assi uniti e suno, oltre a ciò, tangenti coniugate e spigoli del tetraedro di riferimento, permette di seguir con l'intuizione i movimenti del gruppo come meglio non si potrebbe.

N. $\left.10:\left[\begin{array}{ll}1 & 1\end{array}\right)\left(\begin{array}{ll}1 & 1\end{array}\right)\right] \equiv\left[\begin{array}{ll}1 & 1\end{array}\right]$.

Caso corrispondente al simbolo $\overline{(1111)} 11$.

Equazioni differenziali:

$$
\begin{array}{llll}
x_{1}^{\prime}=\sigma_{1} x_{1}, & x_{2}=\sigma_{1} x_{2}, & x_{3}^{\prime}=\sigma_{3} x_{3}, & x_{4}=\sigma_{3} x_{4} \\
\xi_{1}^{\prime}=-\sigma_{1} \xi_{1}, & \check{弓}_{2}^{\prime}=-\sigma_{1} \xi_{2}, & \xi_{3}^{\prime}=-\sigma_{3} \xi_{3}, & \xi_{4}^{\prime}=-\sigma_{3} \xi_{4}^{\prime}
\end{array}
$$

integrali:

$$
\left.\begin{array}{llll}
x_{1}=x_{1}^{0} e^{\sigma_{1} t}, & x_{2}=x_{2}^{0} e^{\sigma_{1} t}, & x_{3}=x_{3}^{0} e^{\sigma_{3} t}, & x_{1}=x_{4}^{0} e^{\sigma_{3} t} \\
\xi_{1}=\xi_{1} e^{-\sigma_{1} t}, & \xi_{2}=\xi_{2}^{0} e^{-\sigma_{1} t}, & \xi_{3}=\xi_{3}^{0} e^{-\sigma_{3} t}, & \xi_{1}=\xi_{4}^{0} e^{-\sigma_{3} t} .
\end{array}\right\}
$$

Gli elementi uniti sono tutti $\mathrm{i}$ punti e tutti i piani delle due rette unite

$$
r \equiv \xi_{1} \cdot \xi_{2} \equiv x_{3} \cdot x_{4}, \quad \rho \equiv \xi_{3} \cdot \xi_{4} \equiv x_{1} \cdot x_{2} .
$$

Le trajettorie sono rette comuni ai piani dei fasci

$$
x_{2}^{0} x_{1}-x_{1}^{0} x_{3}=0, \quad x_{4}^{0} x_{3}-x_{3}^{0} x_{4}=0,
$$

di assi $\rho$ ed $r$, o congiungenti i punti delle punteggiate

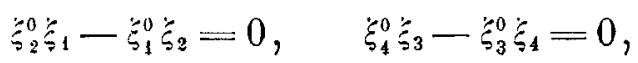


sui sostegni $r$ e $p$. Si hil così una congruenza di $10^{\circ}$ ordine c di $1 .^{\mathrm{a}}$ classe (geschaarte Collineation, prospettiva di 2.a specie di Battaguni, omografia rigata di Segre).

Questo gruppo trasforma in sè ognuno dei due fasci-schiera di quadricho

$$
x_{1} x_{4}+\lambda x_{2} x_{3}=0, \quad x_{1} x_{3}+\lambda^{\prime} x_{2} x_{4}=0 .
$$

Anche il sistema lineare $\boldsymbol{\infty}^{3}$ di quadriche

$$
F(x)=a_{13} x_{1} x_{3}+a_{14} x_{1} x_{4}+a_{23} x_{2} x_{3}+a_{24} x_{2} x_{4}=-=0,
$$

si muta in sè stesso. Le quadriche passano per $r$ c p. Il sistema tangenziale ha la stessa forma.

Essendo poi le equazioni differenziali in coordinate di rette

$$
\begin{gathered}
p_{12}^{\prime}=2 \sigma_{1} p_{12}, \quad p_{34}=2 \sigma_{3} p_{31}, \quad p_{23}^{\prime}=\left(\sigma_{1}+\sigma_{3}\right) p_{23}, \quad p_{14}^{\prime}=\left(\sigma_{1}+\sigma_{3}\right) p_{14} \\
p_{31}^{\prime}=\left(\sigma_{1}+\sigma_{33}\right) p_{31}, \quad p_{24}^{\prime}=\left(\sigma_{1}+\sigma_{3}\right) p_{24},
\end{gathered}
$$

si vede che la serie $\infty^{3}$ di complessi lineari

$$
\alpha_{24} p_{33}+\alpha_{93} p_{14}+\alpha_{14} p_{23}+\alpha_{13} p_{24}=0,
$$

¿̀ unita. A questi complessi appartengono le due rette $r$ e $p$. Ognuno di questi complessi o contiene duo generatrici della quadrica o le contiene tutte. Questo secondo caso avviene sulo quando sia $\alpha_{i j} \equiv a_{h k}$, essendo $i j$ od $h l_{i}$ combinazioni diverse.

All'altro simbolo ( $\left(\begin{array}{llll}1 & 1 & 1 & 1\end{array}\right)\left(\begin{array}{ll}1 & 1)\end{array}\right)$ non corrispondono, nè anche quì, trasformazioni del gruppo. Dorrebbero essere uniti: il fascio-schiera di quadriche

ed il fascio di complessi

$$
x_{1} x_{2}+\mu x_{3} x_{4}=0,
$$

$$
p_{12}+k p_{34}=0 \text {. }
$$

Dovrebbe dunque essere

$$
e^{2 \sigma_{1} t}=e^{i \sigma_{i} t}
$$

ciò che è assurdo perchè $\sigma_{1}$ è divor'so da $\sigma_{3}$.

Al solito, questo caso si presenta in una omografia generale per la quale fosse

$$
\sigma_{3}=-\sigma_{1}
$$

cioc̀ per l'omografia involutoria. Dunque nel gruppo non vi sono omografie involutorie. 
N. $11:[(25)] \equiv\left[\left(\begin{array}{ll}1 & 1\end{array}\right)\right]$.

Equazioni differenziali :

$$
\begin{aligned}
& x_{1}^{\prime}=\sigma_{1} x_{1}+\lambda_{1} x_{3}, \quad x_{2}^{\prime}=\sigma_{1} x_{2}+\lambda_{2} x_{4}, \quad x_{3}^{\prime}=\sigma_{1} x_{3}, \\
& x_{1}^{\prime}=\sigma_{1} x_{4} \\
& \xi_{1}^{\prime}=-\sigma_{1} \xi_{1} \\
& \dddot{\prime}_{2}=-\sigma_{1} \dddot{亏}_{2}, \\
& \left.\xi_{3}^{\prime}=-\sigma_{1} \check{亏}_{3}-\lambda_{1} \check{亏}_{1},\right\} \\
& \check{\zeta}_{4}^{\prime}=-\sigma_{1} \xi_{1}-\lambda_{2} \xi_{2} \text {, }
\end{aligned}
$$

integrali :

$$
\begin{aligned}
x_{1}=\left(x_{1}^{0}+\lambda_{1} x_{3}^{0} t\right) e^{\sigma_{1} t}, & x_{2}=\left(x_{2}^{0}+\lambda_{1} x_{4}^{0} t\right) e^{\sigma_{1} t}, \quad x_{3}=x_{3}^{0} e^{\sigma_{1} t}, \\
x_{1} & =x_{1}^{0} e^{\sigma_{1} t} \\
\xi_{1}=\zeta_{1}^{0} e^{-\sigma_{1} t}, \quad \zeta_{2} & =\xi_{2}^{0} e^{-\sigma_{1} t}, \quad \zeta_{3}=\left(\xi_{3}^{0}-\lambda_{1}\right. \\
\zeta_{4} & =\left(\xi_{4}^{0}-\lambda_{2} \xi_{3}^{0} t\right) e^{-\sigma_{1} t},
\end{aligned}
$$

mentre gli elementi uniti sono tutti i punti ed i piani dell' unica retta

$$
\xi_{1} \cdot \xi_{2}=x_{3} \cdot x_{4}
$$

nella quale coincidono $r$ e $\rho$.

Dalle (2) si possono sopprimere i fattori $e^{\sigma_{1} t}, e^{-\sigma_{1} t}$, ed esse fanno vedere che le trajettorie sono rette appoggiantisi ad $r$, formanti una congruenza a direttrici coincidenti.

Questa è l'omografia riguta speciale di Skgre.

Si trova poi che si trasforma in sè stesso il sistema lineare $\infty^{3}$ di quadriche

$$
F(x) \equiv a_{33} x_{3}^{2}+a_{44} x_{4}^{2}+2 a_{34} x_{3} x_{4}+m\left(\lambda_{2} x_{1} x_{1}-\lambda_{1} x_{2} x_{3}\right)=0,
$$

ed il sistema tangenziale:

$$
\Phi(\xi) \equiv \alpha_{11} \xi_{1}^{2}+\alpha_{22} \xi_{2}^{2}+2 \alpha_{12} \xi_{1} \xi_{2}+\mu\left(\lambda_{2} \xi_{2} \xi_{3}-\lambda_{1} \xi_{1} \xi_{1}\right)=0 .
$$

Queste quadriche $\left(^{*}\right)$ si toccano lungo la retta $r \equiv p$ e si tagliano secondo due rette, mobili, dell'altro sistema. Le trajettorie sono rette di queste quadriche di sistema opposto alla $r \equiv \rho$ : basta far passare $F$ per $x^{0}$ o far toccare $\Phi$ da $\xi_{0}$, perchè la trajettoria-raggio di $x^{0}$ (l'inviluppo-asse di $\xi^{0}$ ) sia posta su $F^{\prime}$ (su $\left.\Phi\right)$.

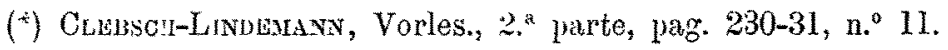


Si trova pure che la serie lineare $\infty^{3}$ di complessi:

$$
\beta\left(\lambda_{1} p_{23}+\lambda_{2} p_{14}\right)+\beta_{24} p_{13}+\beta_{13} p_{24}+\beta_{12} p_{34}=0,
$$

si trasforma in sè.

Il complesso è speciale se

$$
\lambda_{1} \lambda_{2} \beta^{2}-\beta_{13} \beta_{24}=0:
$$

in ogni caso la retta $\xi_{1} \cdot \xi_{2} \equiv r \equiv p$ appartiene al complesso.

N. 12: $\left[\left(\begin{array}{lll}1 & 1 & 1\end{array}\right) 1\right] \equiv\left[\begin{array}{lll}2 & 0\end{array}\right],:\left(\begin{array}{lll}1 & 1 & 1\end{array}\right)\left(\begin{array}{lll}1 & 1 & 1\end{array}\right) !$.

Equazioni differenziali:

$$
\begin{aligned}
& x_{1}^{\prime}=\sigma_{1} x_{1}, \quad x_{2}^{\prime}=\sigma_{1} x_{2}, \quad x_{3}=\sigma_{1} x_{3}, \quad x_{4}^{\prime}=\sigma_{1} x_{4}
\end{aligned}
$$

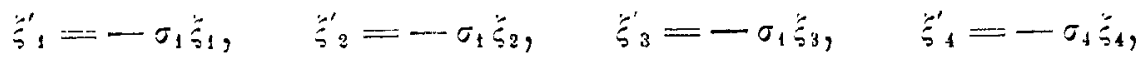

integrali:

$$
\begin{aligned}
& x_{1}=x_{1}^{0} e^{\sigma_{1} t}, \quad x_{2}=x_{2}^{0} e^{\sigma_{1} t}, \quad x_{3}=x_{3}^{0} e^{\sigma_{1} t}, \quad x_{1}=x_{4}^{0} e^{\pi_{4} t}
\end{aligned}
$$

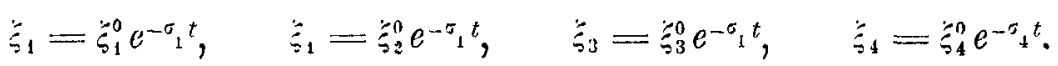

Gli elementi uniti sono:

il punto $\varsigma_{4}=0$ come centro di una stella unita,

il piano $x_{4}=0$ come sostegno di un sistema piano unito.

Questa è l'omologia solida ordinaria (prospettiva di $3 .^{a}$ specie di BaTTALiLIN). Le rette per $\hat{s}_{4}=0$ (centro d'omologia) o le rette in $x_{4}=0$ (piano d'omolugia) sono le trajettorie o gl'inviluppi (caratteristiche di punti o di piani).

Sono quadriche unite tutti $\mathrm{i}$ coni in $\xi_{4}=0$ e tutte le coniche in $x_{4}=0$.

Abbiamo poi le altre equazioni differenziali in coordinate-raggi di rettc

$$
\begin{array}{lll}
p_{23}^{\prime}=2 \sigma_{1} p_{23}, & p_{31}^{\prime}=2 \sigma_{1} p_{31}, & p_{12}^{\prime}=2 \sigma_{1} p_{12} \\
p_{14}^{\prime}=\left(\sigma_{1}+\sigma_{4}\right) p_{14}, & p_{24}^{\prime}=\left(\sigma_{1}+\sigma_{4}\right) p_{24}, & p_{14}^{\prime}=\left(\sigma_{1}+\sigma_{4}\right) p_{34} .
\end{array}
$$

(e dualmente $\pi_{14}^{\prime}=2 \sigma_{1} \pi_{44}, \pi_{23}^{\prime}=\left(\sigma_{1}+\sigma_{14}\right) \pi_{23}$ ecc.).

Onde si vede che $\mathrm{i}$ due complessi speciali

$$
\begin{aligned}
& A \equiv a_{14} p_{23}+a_{94} p_{31}+a_{34} p_{12}=0 \\
& B \equiv b_{23} p_{14}+b_{31} p_{24}+b_{12} p_{34}=0
\end{aligned}
$$


sono uniti. Il primo complesso individua una retta $a$ le cui coordinate-raggi sono $\left(0,0,0, a_{14}, a_{24}, a_{34}\right)$ e che, dunque, passa pel centro $\xi_{4}$ di omologia; la retta $b$ individuata dal secondo complesso ha per coordinate-raggi $\left(b_{23}, b_{31}\right.$, $\left.b_{12}, 0,0,0\right)$ e giace nel piano $x_{4}=0$ di omologia (*). Le rette $a, b$ sono in generale sghembe, perchè in generale non è nullo l'invariante:

$$
a_{14} b_{23}+a_{24} b_{34}+a_{34} b_{12},
$$

e sono direttrici di una congruenza comune ai due complessi speciali. Questa congruenza degenera in un fascio di raggi se $a$ e $b$ si tagliano.

Inoltre due complessi corrispondenti si possono scrivere:

$$
A+B=0, \quad e^{2 \pi_{1} t} A+e^{\left(\sigma_{1}+\sigma_{4}\right) t} B=0 ;
$$

e si vede da ciò ch'essi si tagliano nella congruenza precedente.

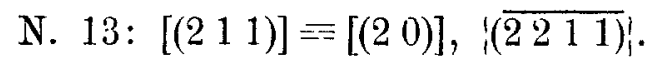

Equazioni differenziali:

$$
\begin{array}{lll}
x_{1}^{\prime}=\sigma_{1} x_{1}+\lambda_{1} x_{4}, & x_{2}^{\prime}=\sigma_{1} x_{2} \quad x_{3}^{\prime}=\sigma_{1} x_{3}, \quad x_{4}^{\prime}=\sigma_{1} x_{4} \\
\xi_{1}^{\prime}=-\sigma_{1} \xi_{1}, & \check{\xi}_{2}^{\prime}=-\sigma_{1} \xi_{2}, \quad \xi_{3}^{\prime}=-\sigma_{1} \xi_{3}, \quad \dddot{\dddot{y}}_{4}^{\prime}=-\sigma_{1} \xi_{4}-\lambda_{1} \xi_{1},
\end{array}
$$

integrali:

$$
\begin{array}{ll}
x_{1}=\left(x_{1}^{0}+\lambda_{1} x_{4}^{0} t\right) e^{\sigma_{1} t}, & x_{2}=x_{2}^{0} e^{\sigma_{1} t}, \quad x_{3}=x_{3}^{0} e^{\sigma_{1} t}, \quad x_{4}=x_{4}^{0} e^{\sigma_{1} t} \\
\varkappa_{1}=\xi_{1}^{0} e^{-\sigma_{1} t}, & \left.\xi_{2}=\xi_{2}^{0} e^{-\sigma_{1} t}, \quad \xi_{3}=\xi_{3}^{0} e^{-\sigma_{1} t}, \quad \zeta_{4}=\left(\xi_{4}^{0}-\lambda_{1} \xi_{1}^{0} t\right) e^{-\sigma_{1} t}\right)
\end{array}
$$

Questa è pure un'omologia, ma quella detta speciale, nella quale il centro ̀̀ $\zeta_{1}=0$ ed il piano ̀̀ $x_{4}=0$ che contiene il centro.

Quadriche unite (coni e coniche) come precedentemente.

Abbiamo poi:

$$
\begin{array}{lll}
p_{12}^{\prime}=2 \sigma_{1} p_{12}-\lambda_{1} p_{24}, & p_{13}^{\prime}=2 \sigma_{1} p_{13}-\lambda_{1} p_{34}, & p_{14}^{\prime}=2 \sigma_{1} p_{14}, \\
p_{24}^{\prime}=2 \sigma_{1} p_{23}, & p^{\prime}{ }_{24}=2 \sigma_{1} p_{24}, & \left.p_{34}^{\prime}=2 \sigma_{14} p_{34} ;\right)
\end{array}
$$

onde il complesso unito, appartenente ad una serie lineare $\infty^{3}$,

Se poi:

$$
B \equiv b_{23} p_{14}+b_{14} p_{23}+b_{31} p_{24}+b_{12} p_{34}=0 \text {. }
$$

$$
C \equiv b_{23} p_{14}+b_{31} p_{24}+b_{12} p_{34}+b_{14} p_{23}+b_{24} p_{34}+b_{34} p_{12}=0
$$

(*) Segre, Ricerche, ecc., pag. 17 . 
¿ l'equazione di un complesso qualunque, quella del suo corrispondente si ottiene, dopo aver eseguita l'integrazione delle (3), sotto la forma:

$$
\Gamma \equiv e^{03_{1} t} \cdot C+e^{2 \sigma_{1}} t \lambda_{1}\left[b_{34} p_{34}-b_{34} p_{24}\right] t=0 \text {. }
$$

Di qui intanto si conclude che il complesso $C$ sarà unito, diverrà, cioè, $B$, se $b_{21}=-b_{34}=0$. In ogni altro caso $\Gamma$ i ma combinazione lineare dei due

$$
C=0 \text { e } \quad b_{24} p_{34}-b_{34} p_{24}=0,
$$

che possiamo scrivere anche, posto $s=\frac{1}{j_{1} t}$, così

$$
\mathbf{l}=s C+b_{24} p_{34}-b_{34} p_{24}=0 \text {. }
$$

Per determinare i complessi spcciali si ha l'equazione

$$
\left(b_{14} b_{23}+b_{34} b_{31}+b_{34} b_{12}\right) s^{2}=0 .
$$

Pel valore doppio $s=0$ si ha dunque il complesso speciale (direttrici infinitamente vicine della congruenza)

$$
b_{24} p_{34}--b_{34} p_{24}=0
$$

che rappresenta una retta le cui coordinate-raggi $q_{i j}$ sono:

$$
q_{12}=b_{24}, \quad q_{31}=-b_{34},
$$

e le altre $q_{i j}$ sono nulle.

Or queste sono le coordinate di una retta per $\zeta_{1}$ nel piano $x_{4}=0$.

L'equazione (4) in $s$ è identica se è nullo l'invariante

$$
b_{14} b_{33}+b_{34} b_{31}+b_{24} b_{12}=0 \text {, }
$$

cioè se il complesso $C$ è speciale saranno allora speciali tutti i complessi $\left.\Gamma^{*}\right)$.

(*) Il dott. Exriques m'invió, durante la redazione della mia Memoria, un suo lavoro pubblicato negli Atti del R. Istituto Veneto: Le superficie con infinile lrasforinazioni projettive in se stesse. Il titolo stesso dinota la differenza del soggetto mio da rucllo dell'egregio dott. Evrirues, malgrado analogie sostanziali. Le superficic esinninate dal dott. ExRIQULS sono degnissime di studio, anche, come mi seriveva l'Autore, dill punto di vista della scomotria diflerenciale (per le assintotiche, per s'sempio). 


\section{§ 3. Gruppi in coordinate non omogenee. Osservazioni.}

Volendo adoperar le coordinate cartesiane, alle quali sole restringerò oramai il mio dire, si ha gran vantaggio nel mandare all' $\infty$ un piano unito corrispondente ad una radice della maggior moltiplicità possibile.

Adottando in questo caso il simbolo

$$
\begin{aligned}
& U f \equiv(x, y, z) \frac{\partial f}{\partial x}+\eta(x, y, z) \frac{\partial f}{\partial y}+\zeta(x, y, z) \frac{\partial f}{\partial z} \\
& \equiv \xi \frac{\partial f}{\partial x}+\eta \frac{\partial f}{\partial y}+\zeta \frac{\partial f}{\partial z},
\end{aligned}
$$

si ha:

$$
\frac{d x}{d t}==5, \quad \frac{d y}{d t}=x, \quad \frac{d z}{d t}=\zeta .
$$

Le funzioni $\zeta, n, \zeta$ si calcolano subito, caso per caso, partendo dalle coordinate non omogenee, nel modo seguente.

Pnngasi per esempio:

$$
x=\frac{x_{4}}{x_{4}}, \quad y=\frac{x_{2}}{x_{4}}, \quad z=\frac{x_{3}}{x_{4}} .
$$

Abbiamo, per le (1) del $\S 1$,

$$
\begin{aligned}
\vdots=\frac{d . x}{d t}= & =\frac{x_{4}\left(a_{11} x_{1}+a_{12} x_{2}+a_{13} x_{3}+a_{14} x_{4}\right)-x_{1}\left(a_{41} x_{1}+a_{42} x_{2}+a_{43} x_{3}+a_{14} x_{4}\right)}{x_{4}^{2}} \\
& =a_{11} x+a_{12} y+a_{13} z+a_{14}-x\left(a_{41} x+a_{42} y+a_{43} z+a_{44}\right) .
\end{aligned}
$$

Simili espressioni si arranno per le $n=\frac{d y}{d t}, \zeta=\frac{d z}{d t}$.

Onde:

$$
\begin{gathered}
U f=\left[a_{11} x+\cdots-x\left(a_{41} x+\cdots\right)\right] \frac{\partial f}{\partial x}+\left[a_{21} x+\cdots-y\left(a_{4} x+\cdots\right)\right] \frac{\partial f}{\partial y} \\
+\left[a_{31} x+\cdots-z\left(a_{41} x+\cdots\right)\right] \frac{\partial f}{\partial z} .
\end{gathered}
$$

Cosicchè dal simbolo

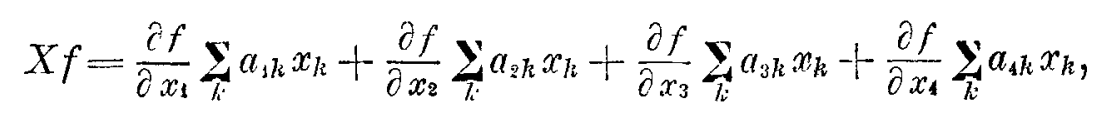

Annali di Matematica, tomo XXII. 
nascerà il simbolo $U f\left({ }^{*}\right)$ sottraendo dai coefficienti di $\frac{\hat{c} f}{\hat{\partial} x_{1}}, \frac{\partial f}{\partial x_{2}}, \frac{\partial f}{\partial x_{3}}$ i prodotti di $x_{1}, x_{2}, x_{3}$ pel coefficiente di $\frac{\partial f}{\partial x_{i}}$, sopprimendo in $X f$ il termine in $\frac{\partial f}{\partial x_{4}}$ e ponendo in ultimo $x_{1}=x, x_{2}=y, x_{3}=z, x_{4}=1$.

Seguono i 13 tipi $U_{i} f(i=1, \ldots, 13)$. Arrerto che $U f$ si può moltiplicare per un fattore costante.

$\left[\begin{array}{llll}1 & 1 & 1 & 1\end{array}\right]$

$$
\begin{gathered}
U_{1} f=\left(\sigma_{1}-\sigma_{4}\right) x \frac{\partial f}{\partial x}+\left(\sigma_{2}-\sigma_{4}\right) y \frac{\partial f}{\partial y}+\left(\sigma_{3}-\sigma_{4}\right) z \frac{\partial f}{\partial z} \\
=\alpha x \frac{\partial f}{\partial x}+\beta y \frac{\partial f}{\partial y}+y z \frac{\partial f}{\partial z} .
\end{gathered}
$$

Le trasformazioni per le quali

cioè:

$$
\alpha=\beta+\%
$$

$$
\sigma_{1}+\sigma_{4}=\sigma_{2}+\sigma_{3} \quad\left[\S 2, n^{\circ} 1\right. \text { equazione (10)], }
$$

mutano in sè stesse le quadriche

quelle per le quali

$$
a_{11} x+2 a_{23} y z=0
$$

$$
\alpha+\beta=0
$$

(i) I'équazione $V f=0$ alle derivate parziali ad un numero qualunque di variahili fu integrata da Hwsse, nol $25^{\circ}$ vol. del Giornale di Crelle, subito dopo che JAcons ebbe integrata, nel vol. 21, l'erquazione differenziale ordinaria a due variabili $x, y$ che porta il stro nome, ed il cui integrale fornisce appunto curve $\|^{r}$ piane. Vedansi, oltre i gia eitati, anche i segnenti lavori o libri:

Imbesaur, tom. 10 del Journal do Liouville, pag. :316-319.

Danboux, Mémoire sur les équalions diffurentielles, ete. nel Bulletin des Scicnees mathématiques, $12{ }^{\text {me }}$ série, tom. 2.", aunce 1878.

Boour, A treatise on differential equations, 1859.

Jordar, Cours dinalyse, tom. 3, pag. 36 e pag. 161 , dove riporta il motodo di Cavary pel sistema d'equazioni difterenzinli ordinarie cui da luogo la $U_{f} f=0$. SERRet, Calcolo integrale.

Fovret, Comptes Rendus, tom. 77, $1 .^{\circ}$ sem., pag. 693 e pag. 1837.

B.traciLin, Sulla geonetria proieltiva, Memoria 3." Acead. di Napoli, 1875. Sut comessi ternarii $(1,1)$, hill., 1880. 
mutano in sè stessi i cilindri

$$
x y-k=0
$$

e finalmente quelle per lo quali

mutano in sè $\mathrm{i}$ coni

$$
\alpha+\beta=2 \%
$$

$$
x y-l i z^{2}=0 .
$$

Giova in questo caso, volendo fare un esempio, supporre prima di tutto a e $\beta$ immaginari coniugati, $\gamma$ reale, poi i piani uniti $x$ ed $y$ coincidenti coi piani ciclici per l'asse $z$.

Scriveremo allora prima $U_{1} f$ e $(2)$ sotto la forma:

$$
\begin{gathered}
U_{1} f=\alpha \frac{\partial f}{\partial \zeta}+\beta n \frac{\partial f}{\partial r_{1}}+\gamma z \frac{\partial f}{\partial z} \\
\vdots n-l z^{2}=0,
\end{gathered}
$$

indi porremo, d'accordo con la (1),

$$
\begin{array}{ll}
\alpha=y+\mu i, & \beta=\gamma-y i \\
\xi=x+y i, & \eta=x-y i .
\end{array}
$$

Le equazioni integrali

diventano:

$$
\xi=\zeta_{0} e^{\alpha t}, \quad \eta=\eta_{0} e^{\beta t}, \quad z=z_{0} e^{i t},
$$

$$
x+i y=\left(x_{0}+i y_{0}\right) e^{(\gamma-1 \mu i) t}, \quad z=z_{0} e^{i t},
$$

(il valore di $n$ essendo coniugato a quello di hanno le

$$
\begin{gathered}
x=\left(x_{0} \cos \mu t-y_{0} \operatorname{sen} \mu \cdot t\right) e^{\gamma t}, \quad y=\left(x_{0} \operatorname{sen} \mu t+y_{0} \cos \mu \cdot t\right) e^{\gamma t} ; \\
z=z_{0} e^{\gamma t} .
\end{gathered}
$$

La (4) con le sostituzioni (6) diviene:

$$
\frac{x^{2}+y^{2}}{z^{2}}=\frac{x_{0}^{2}+y_{0}^{2}}{z_{0}^{2}}=k
$$

determinando $k$ in modo che il cono passi pel punto iniziale $x_{0} y_{0} z_{0}$ : il cono c̀ di rotazione intorno all' asse unito $z$.

La curva (8) giace sul cono (9) ed è la cosidetta spirale conica, elica conica, lossodromia del cono di rotazione, perchè si dimostra facilmente che 
questa curva forma angolo costante così con le generatrici come coi piani meridiani del cono.

Le $x$ ed $y$ delle equazioni (8) rappresentano la proiezione dell' elica conica sul piano perpendicolare all'asse del cono: essa è una spirale logaritmica. L'equazione di questa curva in coordinate polari $\rho$ e $\varphi$, se : è l'angolo costante del raggio vettore e della tangente è:

$$
\rho=p_{0} e^{\left(p-i_{0}\right) \cot } ;
$$

dove $\rho_{0}$ ¿ il raggio vettore corrispondente al valore $\varphi_{0}$.

La prima delle (7) moltiplicata per la coniugata da

$$
\rho=\rho_{0} e^{\gamma t},
$$

e questa parayonata con la (10) porge:

$$
l=\varphi-\varphi_{n}, \quad \cot \tau=\%
$$

la quale ultima equazione dà il significato di $\%$

Volendo trasformare il simbolo $U_{1} f$ nelle nuove coordinate $x, y, \hat{z}$, siccome nol termine $y z \frac{\partial f}{\partial z} x$ cd $y$ non entrano osplicitamente, bastera trusformare il binomio

$$
A f \equiv \alpha \frac{\partial f}{\partial \dot{\partial}}+\beta n \frac{\partial f}{\partial x} .
$$

Esprimendo dalle (6) le $x$ ed $y$ con $\zeta$ ed $n$ si ha:

$$
x=\frac{1}{2}(\xi+n), \quad y=\frac{1}{2 i}(\xi-n),
$$

onde poi

$$
\frac{\partial f}{\partial t}=\frac{1}{2} \frac{\partial f}{\partial x}+\frac{1}{2 i} \frac{\partial f}{\partial y}, \quad \frac{\partial f}{\partial x_{1}}=\frac{1}{2} \frac{\partial f}{\partial x}-\frac{1}{2 i} \frac{\partial f}{\partial y} .
$$

Cosicchè successivamente:

perciò:

$$
\left.\begin{array}{rl}
A f & =\frac{1}{2}(\alpha \xi+\beta \eta) \frac{\partial f}{\partial x}+\frac{1}{2 i}(\alpha \xi-\beta \eta) \frac{\partial f}{\partial y} \\
& =(\gamma x-\mu y) \frac{\partial f}{\partial x}+(y y+\mu x) \frac{\partial f}{\partial y} ;
\end{array}\right\}
$$

$$
U_{1} f=(\gamma x-\mu y) \frac{\partial f}{\partial x}+(y y+u x) \frac{\partial f^{\prime}}{\partial y}+\gamma z \frac{\partial f}{\partial z} .
$$

Questo è il simbolo della "Spiraltransformation" (KLEIN: Einleitung in die 
höhere Geom. II, pag. 221-243; ed anche Liz-Schefrens: Vorles. ïber continuirliche Gruppen, pag. 414), e si può scriverlo così:

$$
U_{1} f=\mu\left(-y \frac{\partial f}{\partial x}+x \frac{\partial f}{\partial y}\right)+\gamma\left(x \frac{\partial f}{\partial x}+y \frac{\partial f}{\partial y}+z \frac{\partial f}{\partial z}\right) .
$$

Il coefficiente di $\mu$ è il simbolo della rotazione intorno all'asse $z$ (dell'asse $x$ verso l'asse $y$ ) ed il coefficiente di $y$ è il simbolo di una omotetia rispetto all' origine (redansi appresso i simboli $U^{\prime}{ }_{6} f, U_{12} f$ ). Perciò la trasformazione si compone di una rotazione intorno ad un asse sommata con una omotetia solida il cui centro è su quell'asse.

$\left[\begin{array}{lll}2 & 1 & 1\end{array}\right]$.

Qui il piano unito doppio i $x_{2}=0$; onde in questo caso giova porre:

$$
z=\frac{x_{1}}{x_{2}}, \quad \xi=\frac{x_{3}}{x_{2}}, \quad y=\frac{x_{4}}{x_{2}},
$$

ed abbiamo:

$$
U_{2} f=\lambda_{1} \frac{\partial f}{\partial z}+\left(\sigma_{3}-\sigma_{2}\right) \frac{\partial f}{\partial \zeta}+\left(\sigma_{4}-\sigma_{2}\right) \eta \frac{\hat{c} f}{\partial r_{1}} .
$$

Un caso molto importante si ha allorchè le radici $\sigma_{3}, \sigma_{4}$ sono immagimaric coniugate $\mathrm{e}$ si ccrcano quelle trasformazioni per le quali

$$
2 \sigma_{2}=\sigma_{3}+\sigma_{4}
$$

nel qual caso (vedi.$^{\circ} 2$ del $\S 2$ ) si mutano in sè $i$ fasci-schiere di coni e di coniche

$$
x_{3} x_{4}+i x_{2}^{2}=0, \quad \xi_{3} \ddot{\xi}_{4}+l \xi_{1}^{2}=0
$$

€, di più, come precedentemente

$$
\xi=x+i y, \quad n=x-i y .
$$

Compatibilmente con (13) poniamo allora:

$$
\sigma_{3}=\sigma_{2}+\mu i, \sigma_{4}=\sigma_{2}-\mu i .
$$

Le equazioni integrali sono in questo caso

$$
\xi=\xi_{0} e^{\mu i t}, \quad \eta=\eta_{0} e^{-p i t}, \quad z=z_{0}+\lambda_{1} t
$$

onde in coordinate $x, y, z$

$$
x=x_{0} \cos \mu t-y_{0} \operatorname{sen} \mu t, \quad y=x_{0} \operatorname{sen} \mu t+y_{0} \operatorname{sen} \mu t, \quad z=z_{0}+\lambda_{1} t \text {. }
$$


Il simbolo $U_{2} f$ si trasforma così:

$$
U_{z} f=\lambda_{1} \frac{\partial f}{\partial z}+\mu i \frac{\partial f}{\partial \hat{\partial}}-\mu i n \frac{\partial f}{\tilde{c} r_{1}},
$$

c, dallar (11) ponondovi $\gamma=0$,

$$
U_{2} f=\mu\left(-y \frac{\partial f}{\partial x}+x \frac{\partial f}{\partial !}\right)+\lambda_{1} \frac{\partial f}{\partial z} .
$$

Le curve (15) sono eliche tracciate sul cilindro retto circolare (14), civè:

$$
\vdots n+\lambda \equiv x^{2}+y^{2}+\lambda \text {, }
$$

dove $\lambda=-\left(x_{0}^{2}+y_{0}^{2}\right)$; ed il simbolo $U_{2} f$ ì quello del movimento clicoidale intorno all' asse $z$, composto della rotazione intorno ad esso o della traslazione lungo esso.

[3 1].

Qui il piano unito triplo is $x_{3}=0$; onde si porrà:

cosicehi:

$$
x=\frac{x_{1}}{x_{3}}, \quad y=\frac{x_{2}}{x_{3}}, \quad z=\frac{x_{4}}{x_{3}},
$$

$$
U_{3} f=\lambda_{11} y \frac{\partial f}{\partial x}+\lambda_{2} \frac{\partial f}{\partial y}+\left(\sigma_{4}-\sigma_{1}\right) z \frac{\partial f}{\partial z} .
$$

[22].

I piani uniti doppi son due $x_{2}=0, x_{4}=0$, cosiechi possiamo porre:

$$
x=\frac{x_{1}}{x_{4}}, \quad y=\frac{x_{2}}{x_{4}}, \quad z=\frac{x_{3}}{x_{4}},
$$

per ottenere poi

$$
U_{1} f=\left[\left(\sigma_{1}-\sigma_{3}\right) x+\lambda_{1} y\right] \frac{\partial f}{\partial x}+\left(\sigma_{1}-\sigma_{3}\right) y \frac{\partial f}{\partial y}+\lambda_{2} \frac{\partial f}{\partial z} .
$$

[4].

Qui manderemo all' $\infty$ il piano $x_{1}=0$ ed arremo:

$$
U_{5} f=\lambda_{1} y \frac{\partial f}{\partial x}+\lambda_{2} z \frac{\partial f}{\partial y}+\lambda_{3} \frac{c t}{\partial z},
$$

pel gruppo le cui trajettorie sono cubiche gobbe esclusivamente. 


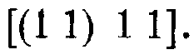

I piani corrispondenti alla radice doppia sono quelli del fascio $\rho \equiv x_{1} \cdot x_{2}$ : porremo perciò:

$$
z=\frac{x_{2}}{x_{1}}, \quad x=\frac{x_{3}}{x_{1}}, \quad y=\frac{x_{4}}{x_{1}},
$$

cosicchè l'asse $\rho$ dei piani uniti cade all' $\infty$ sul piano $z=0$, mentre il lungo dei punti uniti è l'asse delle $z(\equiv x, y=0)$.

Onde avremo qui

$$
U_{i i f} f=\left(\sigma_{3}-\sigma_{1}\right) x \frac{\partial f}{i x}+\left(\sigma_{4}-\sigma_{1}\right) y \frac{\partial f}{\partial y},
$$

per essere:

$$
\frac{d z}{d t}=0 .
$$

Arremo dunque nei piani $z=$ costante delle curve, proiettate in vera forma sul piano $x y$, trajettorie del gruppo piano generale projettivo del tipo:

$$
x \frac{\partial f}{\partial x}+\alpha y \frac{\partial f}{c y},
$$

avendo posto, il che è lecito, $\alpha=\frac{\sigma_{4}-\sigma_{1}}{c_{3}-\sigma_{1}}$.

Le trajettorie del gruppo sono coniche se la $U_{v} f$ si sceglie tra quelle per le quali $\left[\mathbf{n}^{\circ} \quad 6\right.$ equazione (5)]

$$
2 \sigma_{1}=\sigma_{3}+\sigma_{4} .
$$

Allora il gruppo avrà l'espressione semplicissima $(\alpha=-1)$

$$
U^{\prime}{ }^{\prime} f=x \frac{\partial f}{\partial x}-y \frac{\partial f}{\partial y} .
$$

Le proiezioni delle trajettorie sui pinni $z=$ cost. saranno le coniche

$$
x y=x_{0} y_{0},
$$

mentre poi le quadriche $F$ invarianti hanno per equazione:

$$
F=a_{11}+a_{22} z^{2}+2 a_{12} z+2 a_{34} x y=0,
$$

e conterranno le curve (22) se

$$
F_{2}=a_{11}+a_{22} z_{0}^{0}+2 a_{12} z_{0}+2 a_{34} x_{0} y_{0}=0 .
$$


In particolare a questo tipo appartiene il gruppo delle rotazioni intorno all' asse $z$. Soltanto bisogna supporre, d'accordo con (20)

$$
\sigma_{3}=\sigma_{1}+\mu i, \quad \sigma_{4}=\sigma_{1}-\mu i,
$$

e di più, al solito,

$$
x=X+Y i, \quad y=X-Y i .
$$

Allora jl simbolo $U_{6} f$ [redi innanzi noll' equazione (11) pel simbolo $A f$ ponendori $y=0]$ si muta nell' altro:

$$
U^{\prime \prime}{ }_{0} f=-y \frac{\partial f}{\partial x}+x \frac{\partial f}{\partial x},
$$

avendo scritto qui $x, y$ in luogo di $X, Y$.

La $F$ poi diviene:

$$
F=a_{11}+a_{32} z^{2}+2 a_{12} z+2 a_{34}\left(x^{2}+y^{2}\right)=0,
$$

equazione di una quadrica di rotazione intorno all'asse $z$.

$$
\text { [(1 1) 2]. }
$$

Qui si faccin:

$$
x=\frac{x_{4}}{x_{2}}, \quad y=\frac{x_{3}}{x_{9}}, \quad z=\frac{x_{1}}{x_{2}},
$$

e si otterrà:

$$
U_{7} f=\left(\sigma_{3}-\sigma_{1}\right) x \frac{\partial f}{\partial x}+\left[\left(\sigma_{3}-\sigma_{1}\right) y+\lambda_{1} x\right] \frac{\partial f}{\partial y} .
$$

Questo gruppo è quello di quell'omografia nel piano $z=$ cost. [o nella proiezione su $z=0$ ] che ha il simbolo [2 1], possicde, cioè, una retta unita semplice nell' asse $\rho$ all' $\infty$, poi l'unica altra retta unita doppia nell' asse $y$, infine un punto unito semplice nell'origine ed il doppio all' $\infty$ sull' asse $y$.

\section{Ponendo:}

$$
\left.\left[\begin{array}{ll}
2 & 1
\end{array}\right) 1\right] \text {. }
$$

$$
x=\frac{x_{1}}{x_{3}}, \quad y=\frac{x_{4}}{x_{3}}, \quad z=\frac{x_{3}}{x_{3}},
$$

si ha:

$$
U_{8} f=\lambda_{1} \frac{\partial f}{\partial x}+\left(\sigma_{4}-\sigma_{1}\right) y \frac{\partial f}{\partial y}
$$


Osservazione. I due gruppi $U_{7} f$ ed $U_{8} f$ sono rispettivamente riducibili alla forma:

$$
U^{\prime}{ }_{7} f=x \frac{\partial f}{\partial x}+(x+y) \frac{\partial f}{\partial y}, \quad U^{\prime}{ }_{8} f=\frac{\partial f}{\partial x}+y \frac{\partial f}{\partial y}
$$

perchè basta porre:

nel primo $\quad y=Y, \quad \lambda_{1} x=\left(\sigma_{3}-\sigma_{1}\right) X \quad$ e dividere poi per $\left.\sigma_{3}-\sigma_{1}\right)$

nel secondo $y=Y, \quad\left(\sigma_{4}-\sigma_{l}\right) x=\lambda_{1} X$ e dividere poi per $\sigma_{4}-\sigma_{1}$.)

e scrivere di nuovo lettere minuscole.

Or le trasformazioni del tipo $U_{8}^{\prime} f$ hanno per elementi uniti i due punti all' $\infty$ sugli assi delle coordinate la retta all' $\infty$ (come doppia) e l'asse delle $x$ (come semplice).

Adunque tra $i$ due gruppi non v'è che scambio di nomi per gli elementi uniti; perciò i due gruppi, in quanto all'omografia che producono sui piani $z=$ cost. appartengono allo stesso tipo. $\mathrm{E}$ da $U_{i} f$ si deduce $U^{\prime}{ }_{s} f\left({ }^{*}\right)$ ponendo:

$$
x^{\prime}=-\frac{y}{x}, \quad y^{\prime}=\frac{1}{x}
$$

onde:

e perciò:

$$
x=\frac{1}{y^{\prime}}, \quad y=-\frac{x^{\prime}}{y^{\prime}},
$$

$$
U^{\prime}{ }_{7} f=-\frac{\partial f}{\partial x^{\prime}}-y^{\prime} \frac{\partial f}{\partial y^{\prime}} .
$$

La sostituzione, estesa a tutto lo spazio, non è però lineare. Tornando infatti alle coordinate omogenee e chiamando $x_{i}^{\prime}$ le variabili cui si riferisce il gruppo $U_{8} f x_{i}$ quelle cui si riferisce $U_{i}$, si deduce, ritenendo le stesse $z$ :

$$
x^{\prime}=\frac{x_{1}^{\prime}}{x^{\prime}{ }_{3}}=-\frac{x_{3}}{x_{4}}, \quad y^{\prime}=\frac{x_{4}^{\prime}}{x_{3}^{\prime}}=\frac{x_{2}}{x_{4}}, \quad z^{\prime}=\frac{x_{2}^{\prime}}{x_{3}^{\prime}}=z=\frac{x_{1}}{x_{2}},
$$

onde la trasformazione dell'uno nell' altro spazio:

$$
x_{1}^{\prime}: x_{2}^{\prime}: x_{3}^{\prime}: x_{4}^{\prime}=-x_{2} x_{3}: x_{1} x_{4}: x_{2} x: x_{2}^{2},
$$

ch'è quadratica così come la sua inversa.

(*) Vedasi LIE-ŚcheFrers: passim.

Annali di Matematica, tomo XXII. 
Qui abbiamo a porre:

\section{$[(31)]$.}

$$
x=\frac{x_{3}}{x_{1}}, \quad y=\frac{x_{1}}{x_{4}}, \quad z=\frac{x_{2}}{x_{1}},
$$

per ottenere subito:

$$
U_{0} f=\lambda_{2} \frac{\partial f}{\partial x}+\lambda_{1}, x \frac{\partial f}{\partial y} .
$$

Questo gruppo, nei piani $z=$ costante, è del tipo

$$
U_{0}^{\prime} f=\frac{\partial f}{\partial x}+x \frac{\partial f}{\partial y},
$$

corrispondente all'omografia piana [3], con una retta sola unita all' $\infty$ e con un sol punto all' $\infty$ quello dell'asse $y$.

$$
\left.\left[\begin{array}{lll}
(1 & 1
\end{array}\right)\left(\begin{array}{ll}
1 & 1
\end{array}\right)\right]
$$

Fatto:

$$
x=\frac{x_{1}}{x_{4}}, \quad y=\frac{x_{2}}{x_{4}}, \quad z=\frac{x_{3}}{x_{4}},
$$

abbiamo:

$$
U_{10} f=x \frac{\partial f}{\partial x}+y \frac{\partial f}{\partial y}
$$

omotetia nei piani $z=$ cost.

Qui:

$$
[(22)] \text {. }
$$

donde :

$$
x=\frac{x_{1}}{x_{4}}, \quad y=\frac{x_{2}}{x_{4}}, \quad z=\frac{x_{3}}{x_{4}},
$$

$$
U_{11} f=\lambda_{1} z \frac{\partial f}{\partial x_{2}}+\lambda_{2} \frac{\partial f}{\partial y} .
$$

Ponendo:

$$
\left[\begin{array}{lll}
(1 & 1 & 1
\end{array}\right) \text { 1]. }
$$

$$
x=\frac{x_{1}}{x_{4}}, \quad y=\frac{x_{2}}{x_{4}}, \quad z=\frac{x_{3}}{x_{4}},
$$

si trova:

$$
U_{12} f=x \frac{\partial f}{\partial x}+y \frac{\partial f}{\partial y}+z \frac{\partial f}{\partial z} .
$$


Se poi si fa:

$$
x=\frac{x_{1}}{x_{3}}, \quad y=\frac{x_{2}}{x_{3}}, \quad z=\frac{x_{4}}{x_{3}},
$$

si trova:

$$
U^{\prime}{ }_{12} f=z \frac{\partial f}{\partial z},
$$

$U_{12} f$ è il gruppo delle omotetie solide intorno all'origine, $U_{12}^{\prime} f$ è il gruppo delle affinità solide secondo l'asse $z$.

Ponendo:

$$
\text { [(2 } 111)]
$$

si trova:

$$
z=\frac{r_{1}}{x_{4}}, \quad x=\frac{x_{2}}{x_{4}}, \quad y=\frac{x_{3}}{x_{4}},
$$

$$
U_{\mathrm{1} 3} f=\frac{\hat{\sigma} f}{\partial z},
$$

che è il gruppo delle traslazioni parallelamente all' asse $z$.

I primi cinque gruppi sono quelli già discussi del sig. TanNenberg, salvo la diversa scrittura e l'ordine, chè il nostro quarto gruppo è il terzo di quell'autore, e salvo altresì qualche fattore costante o qualche mutamento nelle unità di misura.

Per esempio nel tipo:

$$
U_{4} f=\left[\left(\tau_{1}-\tau_{3}\right) x+\lambda_{1} y\right] \frac{\partial f}{\partial x}+\left(\tau_{1}-\tau_{3}\right) y \frac{\partial f}{\partial y}+\lambda_{2} \frac{\partial f}{\partial z},
$$

basta scrivere:

$$
x=X, \quad y=\frac{\sigma_{1}-\tau_{3}}{\lambda_{1}} Y, \quad z=\frac{\lambda_{2}}{\sigma_{1}-\sigma_{3}} Z,
$$

analogamente a (24), per ottenere il tipo:

$$
(X+Y) \frac{\partial f}{\partial x}+Y \frac{\partial f}{\partial y}+\frac{\partial f}{\partial z},
$$

ch' $’$ il terzo del Tanmenberg.

Roma, lurglio $189+$. 